Article

\title{
Efficient Microwave Assisted Syntheses of 2,5-Diketopiperazines in Aqueous Media ${ }^{\dagger}$
}

\section{Lemuel Pérez-Picaso ${ }^{1}$, Jaime Escalante ${ }^{1}$, Horacio F. Olivo ${ }^{2}$ and María Yolanda Rios ${ }^{1, *}$}

1 Centro de Investigaciones Químicas, Universidad Autónoma del Estado de Morelos, Avenida Universidad 1001, Col. Chamilpa, 62209 Cuernavaca, Morelos, México

2 Medicinal and Natural Products Chemistry, The University of Iowa, Iowa City, IA 52242, USA

$\dagger$ This paper is taken in part from the Ph.D. thesis of Lemuel Pérez-Picaso

* Author to whom correspondence should be addressed; E-mail: myolanda@uaem.mx; Tel. +52 7773297000 ext. 6024; Fax: +52 7773297997

Received: 23 June 2009; in revised form: 21 July 2009 / Accepted: 23 July 2009 /

Published: 31 July 2009

\begin{abstract}
Aqueous in situ one-pot $N$-Boc-deprotection-cyclization of $N \alpha$-Boc-dipeptidyltert-butyl and methyl esters under microwave irradiation afforded 2,5-diketopiperazines (DKPs) in excellent yields. This protocol is rapid, safe, environmentally friendly, and highly efficient, and showed that the tert-butoxy moiety is also an excellent leaving group for these cyclizations.
\end{abstract}

Keywords: 2,5-diketopiperazines; DKPs; cyclic dipeptides; microwave irradiation

\section{Introduction}

Cyclic peptides are a very important family of bioactive compounds easily available both from natural sources (plants, animals or microorganisms) or by means of synthetic methods. Their chemical properties, like the lack of charges at the amine and carboxylic terminal groups and the lack of zwitterionic character, confer to these molecules high lipophilicity [1], and fast membrane absorption in the digestive tract because of their high permeability [2]. The structural rigidity of the cyclic peptides increases its affinity and selectivity toward protein ligands, making their half-lives in vivo much greater than those of linear peptides [3]. Among them, 2,5-diketopiperazines (DKPs, also known as cyclic dipeptides, 2,5-dioxopiperazines, cyclo(dipeptides), or anhydride dipeptides) are the smallest 
cyclopeptides. These peptides are most commonly found as natural products [4], showing antimicrobial [5], antitumoral and antiviral [6], cytotoxic [7], and neuroprotective effects [8], among other activities. Some DKPs are stable to proteolysis (enzymatic degradation), an important feature for their high activity. All these properties make DKPs an interesting group of molecules for the development of new therapeutic agents.

The DKP core derives, chemically and biosynthetically, from folding and head-tail cyclization between $N$ and $C$-terminal amino acids of linear dipeptides [9]. These heterocyclic compounds possess two amide groups (with acceptor-donor properties) with the possibility of including up to four hydrogen bonds [10]. DKPs can be synthesized in solution or in solid phase from commercially available and appropriately protected chiral $\alpha$-amino acids. Their syntheses can be carried out with the appropriate linear dipeptide [11-13], followed by $N$-deprotection and cyclization using either basic (aminolysis of dipeptide ester in methanolic ammonia, Fischer method) [10,14], neutral (aminolysis of dipeptide ester in methanol, autoaminolysis) [15], or acidic conditions (Suzuki method) [16]. Some of these methods variously result in good reaction yields [17], low reaction yields [18], with or without epimerization [19].

Nowadays, microwave heating can be used to obtain good results from previously unsuccessful or low-yielding reactions. Under microwave heating conditions the reaction times can be reduced from days to hours, from hours to minutes, or from minutes to seconds, and the reaction yields can be greatly increased [20,21]. DKPs syntheses using microwave assistance has barely been investigated. Some examples of the use of this methodology are the syntheses of dimeric structures based on intermolecular DKP formation by activation of C-terminal glycine monomers [22], the solvent-free synthesis of DKPs in one-pot deprotection-cyclization of $N$-Boc-dipeptidyl ethyl and methyl esters [23], and the DKPs formation using dipeptide methyl ester hydrochlorides in water in three [24] and two steps [25]. In the present study, we report the syntheses of DKPs using $N \alpha$-Boc-dipeptidyl methyl and tert-butyl esters in water under microwave irradiation. DKPs were obtained in excellent yields without epimerization employing this general and highly efficient protocol.

\section{Results and Discussion}

The formation of DKPs occurs through an intramolecular aminolysis depending largely on the nature and the sequence of the amino acids in solution [11]. Under these conditions, when DKP formation is an undesired reaction, tert-butyl ester protection for the carboxy terminus function is used to prevent ring closure of dipeptides under basic conditions, because it provides a poor leaving group and steric hindrance. Nucleophilic removal of this protecting group with ring formation has been observed only in a few cases [26,27]. We studied the cyclization reaction assisted by microwave heating with the purpose of investigating the possible use of $N \alpha$-Boc-dipeptidyl tert-butyl esters as DKPs precursors, as part of a synthetic strategy toward the cis-DKP fragment of the natural product cyclo[N-(Lys-Phe)-Orn-Val] (1, Figure 1) [28]. We report our findings herein. 
Figure 1. Structure of cyclo[N-(Lys-Phe)-Orn-Val] (1).

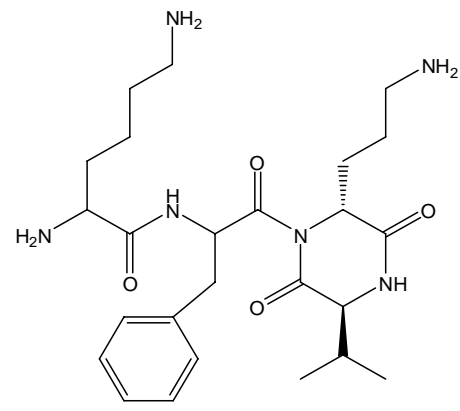

1

In an effort to explore optimized conditions for the cyclization under microwave irradiation, BocOrn(Cbz)-Val-OtBu (2a) was subjected to different reaction variables, such as solvent, temperature $(\mathrm{T})$, irradiation power $(\mathrm{W})$, and exposure time exchange (Table 1). Toluene, toluene-isopropanol (1:1) mixture, and xylene were unsuccessful in the transformation of dipeptide 2a to DKP 3a (entries 1-6). When DMF was used as solvent at $200{ }^{\circ} \mathrm{C}, 300 \mathrm{~W}$, and 5 min reaction time, DKP 3a was obtained in $61 \%$ yield (entry 7). Water resulted a better solvent for the cyclization (entries 8-11). As can be observed in Table 1, with water as solvent, $200{ }^{\circ} \mathrm{C}, 300 \mathrm{~W}$, and $5 \mathrm{~min}$ reaction time resulted in the best conditions, yielding 3a in $89 \%$ yield. However, pressure in the sealed reaction tube increased dramatically due to the generation of $\mathrm{CO}_{2}$ gas, increasing the risk of breaking the reaction vessel. Increasing the temperature and the reaction time did not improve the reaction yield any further (entry 11). The optimized conditions were then used in all cyclizations of $N \alpha$-Boc-dipeptidyl esters 2a$2 \mathbf{l}$ to DKPs 3a-3k.

Table 1. Cyclization of dipeptide 2a to diketopiperazine 3a under microwave irradiation. ${ }^{\mathrm{a}}$

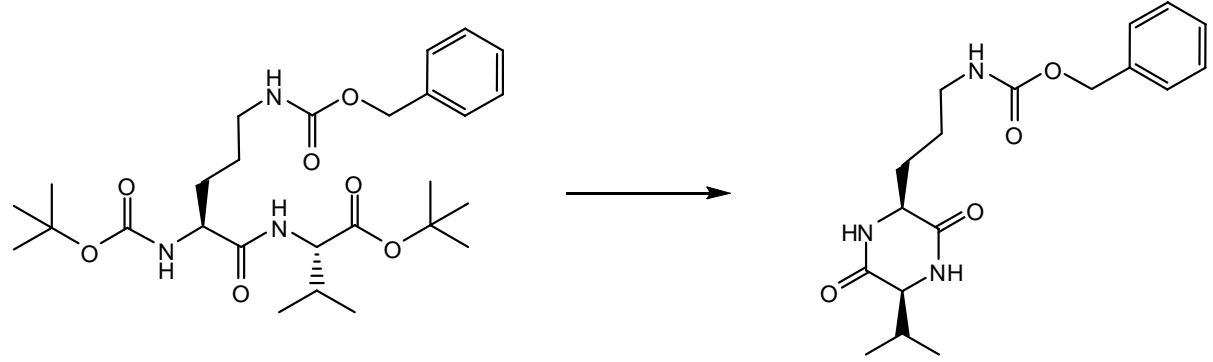

$2 a$

3a

\begin{tabular}{cccccc}
\hline Entry & Solvent & $\mathbf{T}\left({ }^{\circ} \mathbf{C}\right)$ & Power $(\mathbf{W})$ & Time (min) & 3a (\% yield) \\
\hline $\mathbf{1}$ & & 170 & 160 & 10 & $\mathrm{nr}$ \\
$\mathbf{2}$ & 170 & 180 & 10 & $\mathrm{nr}$ \\
$\mathbf{3}$ & toluene & 200 & 250 & 10 & $\mathrm{nr}$ \\
$\mathbf{4}$ & & 200 & 300 & 10 & $\mathrm{nr}$ \\
\hline $\mathbf{5}$ & toluene-isopropanol $(1: 1)$ & 200 & 150 & 10 & $\mathrm{nr}$ \\
\hline $\mathbf{6}$ & xylene & 200 & 300 & 10 & $\mathrm{nr}$ \\
\hline $\mathbf{7}$ & DMF & 200 & 300 & 5 & 61 \\
\hline $\mathbf{8}$ & & 200 & 250 & 1 & 22 \\
$\mathbf{9}$ & & 200 & 250 & 2.5 & 85 \\
$\mathbf{1 0}$ & $\mathrm{H}_{2} \mathrm{O}$ & 200 & 300 & 5 & 89 \\
$\mathbf{1 1}$ & & 250 & 250 & 10 & 86 \\
\hline
\end{tabular}

$\mathrm{nr}=$ no reaction; ${ }^{\mathrm{a}}$ Reactions performed in a monomode microwave CEM Discover apparatus. 
DKPs 3a-3k were synthesized in two steps: dipeptide formation (starting with $N \alpha$-Boc-terminal and $\mathrm{C}-\mathrm{O} t \mathrm{Bu}$ or $\mathrm{C}-\mathrm{OMe}$ amino acids, to obtain the corresponding $N \alpha$-Boc-dipeptidyl esters 2a-2l) and subsequent cyclization (Table 2).

Table 2. Coupling of amino acids and ring closure under microwave irradiation. ${ }^{\mathrm{a}}$

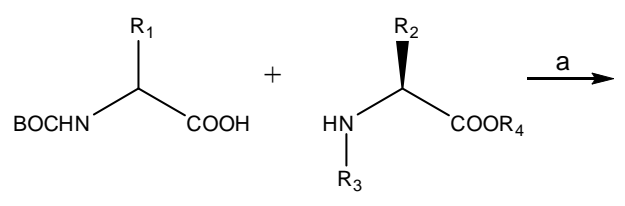<smiles>[R20]C(=O)[C@@H]([R2])N([R3])C(=O)C([R1])NC(=O)O</smiles>

2a-I

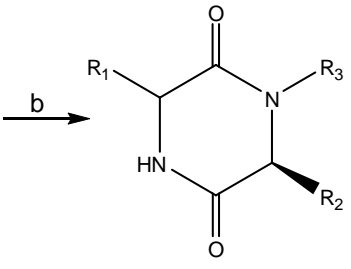

3a-k

(a) EDAC, HOBt, DMAP, TEA $/ \mathrm{CH}_{2} \mathrm{Cl}_{2}, 5{ }^{\circ} \mathrm{C}$, then overnight at rt; (b) $\mathrm{H}_{2} \mathrm{O}(1 \mathrm{~mL}), \mathrm{MW}\left(250{ }^{\circ} \mathrm{C}, 250 \mathrm{~W}\right.$ and $150 \mathrm{psi}$ ) for $10 \mathrm{~min}$.

\begin{tabular}{|c|c|c|c|c|c|c|c|c|}
\hline Entry & $\mathbf{R}_{1}$ & $\mathbf{R}_{2}$ & $\mathbf{R}_{3}$ & $\mathbf{R}_{4}$ & Compound & Yield (\%) & Compound & Yield (\%) \\
\hline 1 & (S)-Propyl-NH-Cbz & Isopropyl & $\mathrm{H}$ & $\mathrm{O} t \mathrm{Bu}$ & $2 a$ & 94 & 3a & $86^{\mathrm{b}}$ \\
\hline 2 & (R)-Propyl- $N \mathrm{H}-\mathrm{Cbz}$ & Isopropyl & $\mathrm{H}$ & $\mathrm{O} t \mathrm{Bu}$ & 2b & 98 & $3 \mathbf{b}$ & $99^{\mathrm{b}}$ \\
\hline 3 & Propyl- $N \mathrm{H}-\mathrm{Cbz}$ & Benzyl & $\mathrm{H}$ & $\mathrm{O} t \mathrm{Bu}$ & 2c & 91 & 3c & $95^{\mathrm{b}}$ \\
\hline 4 & Propyl- $N H-C b z$ & Benzyl & $\mathrm{H}$ & $\mathrm{OMe}$ & 2d & 77 & $3 c$ & $99^{\mathrm{b}}$ \\
\hline 5 & $\mathrm{H}$ & Benzyl & $\mathrm{H}$ & $\mathrm{O} t \mathrm{Bu}$ & $2 \mathbf{e}$ & 98 & $3 \mathbf{e}$ & $99^{\mathrm{b}}$ \\
\hline 6 & Benzyl & Benzyl & $\mathrm{H}$ & $\mathrm{O} t \mathrm{Bu}$ & $2 f$ & 98 & $3 f$ & $96^{\mathrm{b}}$ \\
\hline 7 & Isopropyl & Benzyl & $\mathrm{H}$ & $\mathrm{O} t \mathrm{Bu}$ & $2 g$ & 99 & $3 g$ & $99^{\mathrm{b}}$ \\
\hline 8 & $\mathrm{H}$ & Isopropyl & $\mathrm{H}$ & $\mathrm{O} t \mathrm{Bu}$ & $2 h$ & 73 & $3 \mathbf{h}$ & $93^{\mathrm{b}}$ \\
\hline 9 & Benzyl & Isopropyl & $\mathrm{H}$ & $\mathrm{O} t \mathrm{Bu}$ & $2 \mathbf{i}$ & 80 & $3 g$ & $73^{\mathrm{b}}$ \\
\hline 10 & Isopropyl & Isopropyl & $\mathrm{H}$ & $\mathrm{O} t \mathrm{Bu}$ & $2 \mathbf{j}$ & 83 & $3 \mathbf{j}$ & $99^{\mathrm{b}}$ \\
\hline 11 & Benzyl & $\mathrm{H}$ & $\mathrm{CH}_{3}$ & $\mathrm{O} t \mathrm{Bu}$ & $2 \mathbf{k}$ & 85 & $3 \mathbf{k}$ & $84^{\mathrm{c}}$ \\
\hline 12 & Benzyl & $\mathrm{H}$ & $\mathrm{CH}_{3}$ & $\mathrm{OMe}$ & 2l & 82 & $3 \mathbf{k}$ & $99^{\mathrm{c}}$ \\
\hline
\end{tabular}

Data in Table 2 clearly shows that the cyclization step assisted by microwave heating yielded DKP compounds 3a-3k in excellent yields. Because $N$-Boc protecting groups are unstable at temperatures higher than $90{ }^{\circ} \mathrm{C}$, microwave irradiation should deprotect the amines facilitating the spontaneous intramolecular aminolysis [29]. Nature and size of alkyl group $\left(\mathrm{R}_{1}\right)$ on $\mathrm{C} \alpha$ of $N \alpha$-Boc amino acid residue have no effect on the course and reaction yield when the $\mathrm{C}$ terminus amino acid is phenylalanine (entries 3-7). However, they do influence the cyclization yield when the amino acid at $\mathrm{C}$ terminus is valine (entries $1,8-10$ ). When valine is present, the amino acid residue sequence is important on the cyclization yield (entries 7 and 9) because $\beta$-branched amino acid at $\mathrm{C}$ terminus $(\mathrm{C} \alpha$ isopropyl group) exerts a bulky effect on ring closure, diminishing the reaction yield (99 vs $73 \%$ ). Entries 1 and 3 where a benzyl group replaced an isopropyl one corroborate this steric effect as the reaction yield diminished from 95 to $86 \%$. The leaving groups $\mathrm{O} t \mathrm{Bu}$ and $\mathrm{OMe}$ (ester group nature, entries 3,4 and 11,12) have no effect on course and reaction yield, as corroborated by the excellent yields obtained for compounds $\mathbf{3 a}-\mathbf{3} \mathbf{h}$ and $\mathbf{3} \mathbf{j}-\mathbf{3 k}$. Optical rotation values of compounds $\mathbf{3 a}-\mathbf{3} \mathbf{k}$ and their comparison with literature data showed that cyclization reaction proceeded without $\mathrm{C} \alpha$ chiral center epimerization of the amino acid residues. The cyclization of different $N \alpha$-Boc-dipeptidyl esters (even $\mathrm{O} t$ - $\mathrm{Bu}$ ) in water assisted by microwaves is a rapid, secure, environmentally friendly and highly efficient method to produce $c i s$-DKPs with high optical purity. This reaction conditions are compatible with the 
presence of $\mathrm{Cbz}$ protecting groups. This protocol was used to obtain the trans-DKP fragment $\mathbf{3 b}$ of compound 1, which was synthesized in quantitative yield starting of Boc-D-Orn(Cbz)-Val-OtBu (2b, entry 2).

The structures of compounds $\mathbf{2 a - 2 l}$ and 3a-3k were established on the basis of the analysis of their spectroscopic data. For compounds 2a-2l, carbamate, amide, and ester groups showed IR absorptions at 3,364-3,283, 2,979-2,972 and 1,690-1,665, and 1,744-1,712 $\mathrm{cm}^{-1}$, respectively; Carbonyl amide, ester, $N$-Boc-carbamate, and $N \delta$-Cbz-carbamate groups showed ${ }^{13} \mathrm{C}-\mathrm{NMR}$ resonance signals at $\delta 172.35-169.07, \delta 172.10-167.89, \delta 157.09-155.07$, and $\delta 156.98-156.94$. Carbamate and amide groups in compounds 3a-3k produce IR absorptions at 3,440-3,426, and 2,977-2,925 and 1,678$1,664 \mathrm{~cm}^{-1}$, respectively. Amide groups showed ${ }^{13} \mathrm{C}-\mathrm{NMR}$ resonance signals at $\delta 167.83-166.00$, and $N \delta$-Cbz-carbamate carbonyls give resonance signals in the $\delta 156.06-155.79$ range.

\section{Experimental}

\subsection{General procedures}

Reactions were performed in sealed vessels in a monomode microwave CEM Discover apparatus and the temperature was evaluated by infrared. Melting points were obtained in a Fisher Johns melting point apparatus and are uncorrected. Infrared (IR) spectra were obtained in $\mathrm{KBr}$ on a Bruker Vector 22 IR spectrometer. Optical rotations were measured with sodium light (unless otherwise specified) on a Perkin-Elmer $341 \mathrm{MC}$ polarimeter. ${ }^{1} \mathrm{H}$ - and ${ }^{13} \mathrm{C}$-NMR spectra were recorded on a Varian Unity 400 spectrometer at $400 \mathrm{MHz}$ for ${ }^{1} \mathrm{H}-\mathrm{NMR},{ }^{1} \mathrm{H}-{ }^{1} \mathrm{H} \mathrm{COSY}$, HSQC, and $\mathrm{HMBC}$, and at $100 \mathrm{MHz}$ for ${ }^{13} \mathrm{C}-$ $\mathrm{NMR}$, using $\mathrm{CDCl}_{3}$ or DMSO as solvents, as indicated. Chemical shifts are reported in ppm $(\delta)$ relative to the TMS signal. FAB ${ }^{+} \mathrm{MS}$, and $\mathrm{HRFAB}^{+} \mathrm{MS}$ were recorded on a JEOL JMStation-JM 700 mass spectrometer at $70 \mathrm{eV}$ in a matrix of glycerol. Flash column chromatography (FCC) and analytical thin-layer chromatography (TLC) were performed using silica gel 230-400 mesh and pre-coated silica gel 60 F254 Merck plates, respectively. Boc-Phe-OH, Boc-Val-OH, Boc-Orn(Cbz)$\mathrm{OH}$, Boc-Gly-OH, Sar-OMe, Sar-OtBu, Phe-OMe, Phe-O $t \mathrm{Bu}, \mathrm{Val}-\mathrm{O} t \mathrm{Bu}$, EDAC, TEA, and DMAP were obtained from Aldrich, HOBt was obtained from ANASPEC, and all chemicals were used without further purification.

\subsection{General procedure for the syntheses of dipeptides $\mathbf{2} \mathbf{a}-\mathbf{2} \mathbf{j}$}

A mixture of Boc-amino acid $(1 \mathrm{mmol})$, amino ester hydrochloride $(1 \mathrm{mmol})$, EDAC $(1.5 \mathrm{mmol})$, $\mathrm{HOBt}(1 \mathrm{mmol})$ and DMAP $(0.1 \mathrm{mmol})$ were dissolved in dry $\mathrm{CH}_{2} \mathrm{Cl}_{2}(10 \mathrm{~mL})$. Mixture was cooled to $5{ }^{\circ} \mathrm{C}$ and then TEA $(1 \mathrm{mmol})$ was added. Reaction was stirred at $5{ }^{\circ} \mathrm{C}$ for further $30 \mathrm{~min}$, then allowed to warm up to room temperature and stirred overnight. Reaction mixture was treated with sat. $\mathrm{NH}_{4} \mathrm{Cl}$ soln. $(20 \mathrm{~mL})$. The organic phase was separated and the aqueous layer was extracted with $\mathrm{CH}_{2} \mathrm{Cl}_{2}$ $(3 \times 15 \mathrm{~mL})$. Combined organic layers were washed with brine $(2 \times 15 \mathrm{~mL})$ and with water $(2 \times 15 \mathrm{~mL})$ and dried over $\mathrm{Na}_{2} \mathrm{SO}_{4}$. Solvent was removed under vacuum. The residue was purified by FCC. 


\subsection{General procedure for the syntheses of dipeptides $\mathbf{2 k - 2 \mathbf { l }}$}

A mixture of Boc-amino acid $(2.4 \mathrm{mmol})$, amino ester hydrochloride $(2 \mathrm{mmol})$, EDAC (2.4 mmol), HOBt $(2.4 \mathrm{mmol})$ and DMAP $(0.1 \mathrm{mmol})$ were dissolved in dry $\mathrm{CH}_{2} \mathrm{Cl}_{2}(5 \mathrm{~mL})$. Mixture was cooled to $5{ }^{\circ} \mathrm{C}$ and then TEA $(2.4 \mathrm{mmol})$ was added. Reaction was stirred at $5{ }^{\circ} \mathrm{C}$ for further $30 \mathrm{~min}$, then allowed to warm up to room temperature and stirred for two days. Reaction mixture was treated with sat. $\mathrm{NH}_{4} \mathrm{Cl}$ soln. $(20 \mathrm{~mL})$. The organic phase was separated and the aqueous layer was extracted with $\mathrm{CH}_{2} \mathrm{Cl}_{2}(3 \times 15 \mathrm{~mL})$. Combined organic layers were washed with brine $(2 \times 15 \mathrm{~mL})$ and with water $(2 \times 15 \mathrm{~mL})$ and dried over $\mathrm{Na}_{2} \mathrm{SO}_{4}$. Solvent was removed under vacuum. The residue was purified by FCC.

Boc-Orn(Cbz)-Val-OtBu (2a): Colorless syrup; $[\alpha]+8.5$ (c 1.09, $\mathrm{CHCl}_{3}$ ); IR: 3,336, 2,973, 2,935, 2,878, 1,712, 1,666, 1,532, 1,454, 1,368, 1,254, 1,162, $1,018 \mathrm{~cm}^{-1} ;{ }^{1} \mathrm{H}-\mathrm{NMR}\left(\mathrm{CDCl}_{3}\right) \delta$ 7.28-7.20 (5H, m, Ar), 6.85 (1H, bs, NH-Val), 5.29 (1H, bs, NH-Orn), 5.18 (1H, bs, NHס-Orn), 5.04 (1H, d, $J=12.4$ $\left.\mathrm{Hz}, \mathrm{CH}_{2}-\mathrm{Cbz}\right), 5.01\left(1 \mathrm{H}, \mathrm{d}, J=12.4 \mathrm{~Hz}, \mathrm{CH}_{2}-\mathrm{Cbz}\right), 4.33(1 \mathrm{H}, \mathrm{dd}, J=9.2,4.8 \mathrm{~Hz}, \mathrm{H \alpha}-\mathrm{Val}), 4.24(1 \mathrm{H}$,

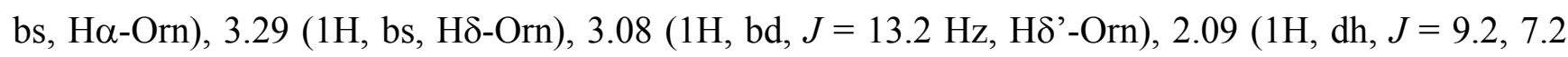

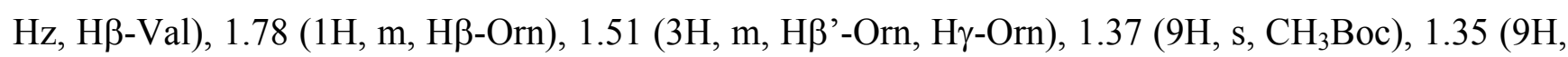
$\left.\mathrm{s}, \mathrm{CH}_{3} t \mathrm{Bu}\right), 0.86(3 \mathrm{H}, \mathrm{d}, J=7.2 \mathrm{~Hz}, \mathrm{H} \gamma$-Val $), 0.84(3 \mathrm{H}, \mathrm{d}, J=7.2 \mathrm{~Hz}, \mathrm{H} \gamma \cdot-\mathrm{Val}) ;{ }^{13} \mathrm{C}-\mathrm{NMR}\left(\mathrm{CDCl}_{3}\right) \delta$ 172.26 (s, CO-Orn), 170.84 (s, CO-Val), 156.97 (s, CO-Cbz), 155.79 (s, CO-Boc), 136.63 (s, Ar), 128.49 (d, Ar), 128.11 (d, Ar), 128.07 (d, Ar), 81.87 (s, C-Boc), 79.89 (s, C-tBu), 66.80 (t, CH${ }_{2}-\mathrm{Cbz}$ ), 57.65 (d, C $\alpha$-Val), 53.41 (d, C $\alpha$-Orn), 40.02 (t, C $\delta$-Orn), 31.28 (d, C $\beta$-Val), 30.10 (t, C $\beta-O r n), 28.50$

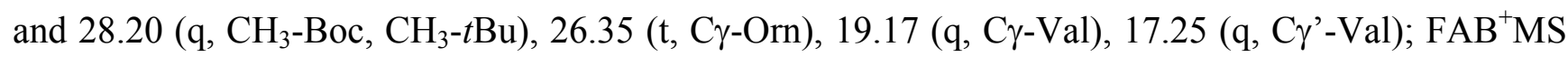
$m / z: 522(53)[\mathrm{M}+\mathrm{H}]^{+}, 466(11)\left[\mathrm{M}+\mathrm{H}-\mathrm{C}_{4} \mathrm{H}_{8}\right]^{+}, 422(13)\left[\mathrm{M}+\mathrm{H}-\mathrm{C}_{5} \mathrm{H}_{8} \mathrm{O}_{2}\right]^{+}, 414$ (15) $[\mathrm{M}-$ $\left.\mathrm{C}_{7} \mathrm{H}_{7} \mathrm{O}\right]^{+}, 366(100)\left[\mathrm{M}+\mathrm{H}-\mathrm{C}_{5} \mathrm{H}_{8} \mathrm{O}_{2}-\mathrm{C}_{4} \mathrm{H}_{8}\right]^{+}, 258(13), 213(20), 91(100)\left[\mathrm{C}_{7} \mathrm{H}_{7}\right]^{+}, 57(44)\left[\mathrm{C}_{4} \mathrm{H}_{9}\right]^{+}$; $\mathrm{HRFAB}^{+} \mathrm{MS}$ : observed $522.3176[\mathrm{M}+\mathrm{H}]^{+}$, (calcd. for $\mathrm{C}_{27} \mathrm{H}_{44} \mathrm{~N}_{3} \mathrm{O}_{7}, 522.3179$ ).

Boc-D-Orn (Cbz)-Val-OtBu (2b): Colorless syrup; [ $\alpha]+15.7$ (c 1.04, $\mathrm{CHCl}_{3}$ ); IR: 3,339, 2,973, 2,935, 2,877, 1,712, 1,666, 1,525, 1,456, 1,391, 1,368, 1,254, 1,165, 1,022, 738, $699 \mathrm{~cm}^{-1} ;{ }^{1} \mathrm{H}-\mathrm{NMR}\left(\mathrm{CDCl}_{3}\right)$ $\delta$ 7.36-7.25 (5H, m, Ar), $6.89(1 \mathrm{H}, \mathrm{bs}, \mathrm{NH}-\mathrm{Val}), 5.36(1 \mathrm{H}, \mathrm{bd}, J=6.6 \mathrm{~Hz}, \mathrm{NH}-\mathrm{Orn}), 5.25(1 \mathrm{H}, \mathrm{bs}$,

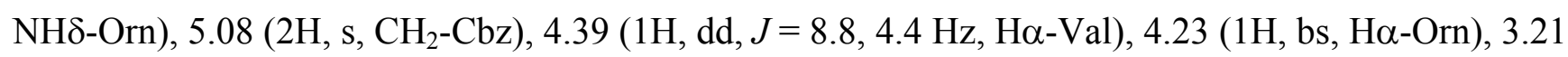

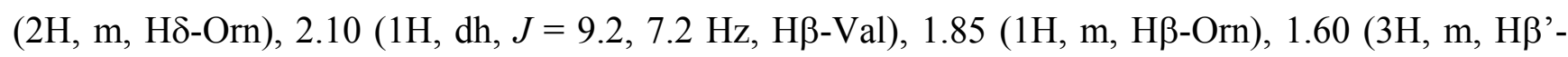

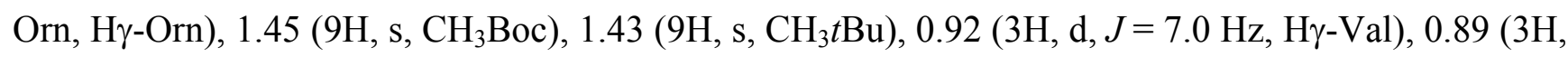
$\left.\mathrm{d}, J=7.0 \mathrm{~Hz}, \mathrm{H} \gamma^{\prime}-\mathrm{Val}\right) ;{ }^{13} \mathrm{C}-\mathrm{NMR}\left(\mathrm{CDCl}_{3}\right) \delta 172.06$ (s, CO-Orn), 170.78 (s, CO-Val), 156.74 (s, COCbz), 155.71 (s, CO-Boc), 136.69 (s, Ar), 128.50 (d, Ar), 128.06 (d, Ar), 82.00 (s, C-Boc), 80.06 (s, C-

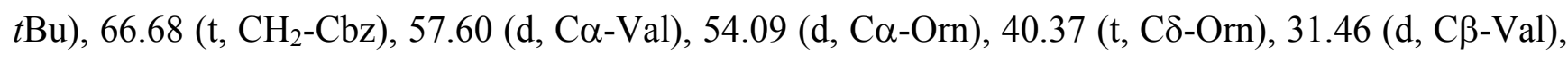
30.07 (t, $\mathrm{C} \beta-O r n$ ), 28.47 and 28.19 (q, $\mathrm{CH}_{3}-t \mathrm{Bu}, \mathrm{CH}_{3}-\mathrm{Bu}$ ), 26.39 (t, $\mathrm{C} \gamma-\mathrm{Orn}$ ), 19.11 (q, $\left.\mathrm{C} \gamma-\mathrm{Val}\right), 17.77$ (q, C $\left.\gamma^{\prime}-V a l\right) ; \mathrm{FAB}^{+} \mathrm{MS} m / z: 522(9)[\mathrm{M}+\mathrm{H}]^{+}, 466$ (3) $\left[\mathrm{M}+\mathrm{H}-\mathrm{C}_{4} \mathrm{H}_{8}\right]^{+}, 422(4)\left[\mathrm{M}+\mathrm{H}-\mathrm{C}_{5} \mathrm{H}_{8} \mathrm{O}_{2}\right]^{+}$, 410 (9), 366 (39) $\left[\mathrm{M}+\mathrm{H}-\mathrm{C}_{9} \mathrm{H}_{17} \mathrm{O}_{2}\right]^{+}, 258(8), 213(15), 91(100)\left[\mathrm{C}_{7} \mathrm{H}_{7}\right]^{+}, 72(28)\left[\mathrm{C}_{4} \mathrm{H}_{8} \mathrm{O}\right]^{+}, 57(38)$ $\left[\mathrm{C}_{4} \mathrm{H}_{9}\right]^{+}$; HRFAB ${ }^{+} \mathrm{MS}$ : observed $522.3176[\mathrm{M}+\mathrm{H}]^{+}$, (calcd. for $\mathrm{C}_{27} \mathrm{H}_{44} \mathrm{~N}_{3} \mathrm{O}_{7}, 522.3179$ ).

Boc-Orn(Cbz)-Phe-OtBu (2c): White solid; Mp 104-105 ${ }^{\circ} \mathrm{C}\left\{\right.$ lit [30] $\left.102{ }^{\circ} \mathrm{C}\right\} ;[\alpha]+25.8$ (c 1.01, $\mathrm{CHCl}_{3}$ ); IR: 3,364, 2,977, 2,880, 1,732, 1,690, 1,668, 1,524, 1,452, 1,368, 1,280, 1,240, 1,164, 1,027 $\mathrm{cm}^{-1} ;{ }^{1} \mathrm{H}-\mathrm{NMR}\left(\mathrm{CDCl}_{3}\right): \delta$ 7.35-7.15 $(10 \mathrm{H}, \mathrm{m}, \mathrm{Ar}), 6.82(1 \mathrm{H}, \mathrm{d}, J=6.8 \mathrm{~Hz}, \mathrm{NH}-\mathrm{Phe}), 5.18(1 \mathrm{H}, \mathrm{d}$, 
$J=6.8 \mathrm{~Hz}, \mathrm{NH}-\mathrm{Orn}), 5.06\left(2 \mathrm{H}, \mathrm{d}, J=12.8 \mathrm{~Hz}, \mathrm{CH}_{2}-\mathrm{Cbz}, \mathrm{NH} \delta-\mathrm{Orn}\right), 5.02\left(1 \mathrm{H}, \mathrm{d}, J=12.8 \mathrm{~Hz}, \mathrm{CH}_{2^{-}}\right.$

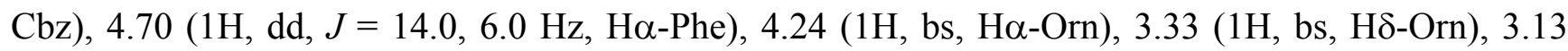
(1H, m, H $\left.\delta^{\prime}-O r n\right), 3.08$ (1H, dd, $J=13.6,14.0 \mathrm{~Hz}, \mathrm{H} \beta$-Phe), 3.04 (1H, dd, $J=13.6,6.0 \mathrm{~Hz}, \mathrm{H} \beta$ '-Phe),

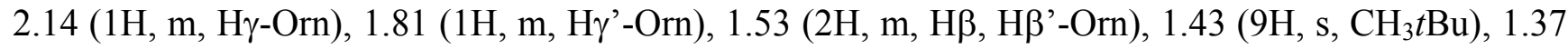
(9H, s, $\left.\mathrm{CH}_{3} \mathrm{Boc}\right) ;{ }^{13} \mathrm{C}-\mathrm{NMR}\left(\mathrm{CDCl}_{3}\right): \delta 171.80$ (s, CO-Orn), 170.48 (s, CO-Phe), 156.94 (s, CO-Cbz), 155.66 (s, CO-Boc), 136.61 (s, Ar-Cbz), 136.23 (s, Ar), 129.58 (d, Ar), 128.58 (d, Ar), 128.49 (d, Ar), 128.16 (d, Ar), 127.03 (d, Ar), 82.41 (s, C-Boc), 80.07 (s, C-tBu), 66.88 (t, CH $\mathrm{CH}_{2}-\mathrm{Cbz}$ ), 53.81 (d, C $\alpha-$

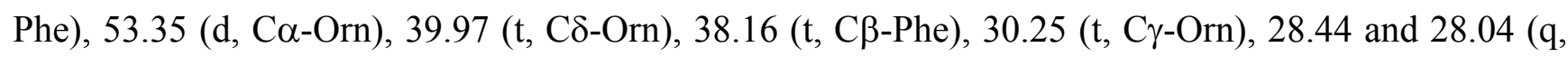
$\mathrm{CH}_{3}-\mathrm{Bu}, \mathrm{CH}_{3}-\mathrm{Bu}$ ), 26.27 (t, $\left.\mathrm{C} \beta-O r n\right) ; \mathrm{FAB}^{+} \mathrm{MS} m / z: 570(56)[\mathrm{M}+\mathrm{H}]^{+}, 556(9)\left[\mathrm{M}+\mathrm{H}-\mathrm{CH}_{2}\right]^{+}, 514$ (8) $\left[\mathrm{M}+\mathrm{H}-\mathrm{C}_{4} \mathrm{H}_{8}\right]^{+}, 470(56)\left[\mathrm{M}+\mathrm{H}-\mathrm{C}_{5} \mathrm{H}_{8} \mathrm{O}_{2}\right]^{+}, 414$ (90) $\left[\mathrm{M}+\mathrm{H}-\mathrm{C}_{5} \mathrm{H}_{8} \mathrm{O}_{2}-\mathrm{C}_{4} \mathrm{H}_{8}\right]^{+}, 306$ (12) $[\mathrm{M}+$ $\left.\mathrm{H}-\mathrm{C}_{14} \mathrm{H}_{19} \mathrm{NO}_{3}-\mathrm{CH}_{3}\right]^{+}, 261$ (17), 204 (14), 154 (27), 120 (27), 91 (100) $\left[\mathrm{C}_{7} \mathrm{H}_{7}\right]^{+}, 57$ (44) $\left[\mathrm{C}_{4} \mathrm{H}_{9}\right]^{+}$; $\mathrm{HRFAB}^{+} \mathrm{MS}$ : observed $570.3152[\mathrm{M}+\mathrm{H}]^{+}$, (calcd. for $\left.\mathrm{C}_{31} \mathrm{H}_{44} \mathrm{~N}_{3} \mathrm{O}_{7}, 570.3179\right)$.

Boc-Orn(Cbz)-Phe-OMe (2d): White solid; Mp 118-120 ${ }^{\circ} \mathrm{C}\left\{\right.$ lit [31] 106-118 $\left.{ }^{\circ} \mathrm{C}\right\} ;[\alpha]-7.5$ (c 1.01, $\mathrm{MeOH})\{$ lit [31] - 7.6 (c 1.1, MeOH)\}; IR: 3,339, 2,974, 2,939, 2,876, 1,743, 1,677, 1,531, 1,449, 1,369, 1,278, 1,249, 1,172, 1,032 $\mathrm{cm}^{-1} ;{ }^{1} \mathrm{H}-\mathrm{NMR}\left(\mathrm{CDCl}_{3}\right) \delta$ 7.36-7.10 (10H, m, Ar), $7.00(1 \mathrm{H}, \mathrm{d}$, $J=7.6 \mathrm{~Hz}, \mathrm{NH}-\mathrm{Phe}), 5.24(1 \mathrm{H}, \mathrm{d}, J=8.0 \mathrm{~Hz}, \mathrm{NH}-\mathrm{Orn}), 5.12(1 \mathrm{H}, \mathrm{t}, J=6.0 \mathrm{~Hz}, \mathrm{NH} \delta-O r n), 5.03(1 \mathrm{H}$, $\left.\mathrm{d}, J=12.8 \mathrm{~Hz}, \mathrm{CH}_{2}-\mathrm{Cbz}\right), 4.99\left(1 \mathrm{H}, \mathrm{d}, J=12.8 \mathrm{~Hz}, \mathrm{CH}_{2}-\mathrm{Cbz}\right), 4.83(1 \mathrm{H}, \mathrm{dd}, J=13.2,6.4 \mathrm{~Hz}, \mathrm{H \alpha}-$

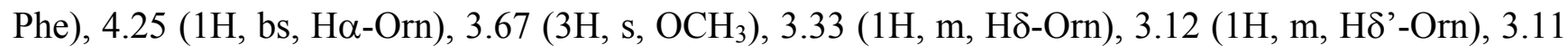
(1H, dd, $J=13.6,5.6 \mathrm{~Hz}, \mathrm{H} \beta-\mathrm{Phe}), 3.05$ (1H, dd, $J=13.6,6.8 \mathrm{~Hz}, \mathrm{H} \beta$ '-Phe), 1.78 (1H, m, H $\gamma$-Orn), $1.52\left(3 \mathrm{H}, \mathrm{m}, \mathrm{H} \beta, \mathrm{H} \beta, \mathrm{H} \gamma\right.$-Orn), $1.42\left(9 \mathrm{H}, \mathrm{s}, \mathrm{CH}_{3} \mathrm{Boc}\right) ;{ }^{13} \mathrm{C}-\mathrm{NMR}\left(\mathrm{CDCl}_{3}\right) \delta 172.10$ (s, CO-Phe), 171.91 (s, CO-Orn), 156.98 (s, CO-Cbz), 155.68 (s, CO-Boc), 136.58 (s, Ar-Cbz), 135.95 (s, Ar), 129.31 (d, Ar), 128.64 (d, Ar), 128.55 (d, Ar), 128.14 (d, Ar), 127.14 (d, Ar), 80.04 (s, C-Boc), 66.82

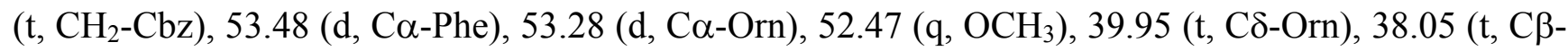

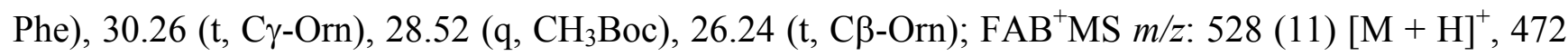
(7) $\left[\mathrm{M}+\mathrm{H}-\mathrm{C}_{4} \mathrm{H}_{9}\right]^{+}, 428(62)\left[\mathrm{M}+\mathrm{H}-\mathrm{C}_{5} \mathrm{H}_{8} \mathrm{O}_{2}\right]^{+}, 320$ (9) $\left[\mathrm{C}_{17} \mathrm{H}_{24} \mathrm{~N}_{2} \mathrm{O}_{4}\right]^{+}, 275(10), 180$ (19) $\left[\mathrm{C}_{10} \mathrm{H}_{14} \mathrm{NO}_{2}\right]^{+}, 120(31)\left[\mathrm{C}_{8} \mathrm{H}_{10} \mathrm{~N}\right]^{+}, 91(100)\left[\mathrm{C}_{7} \mathrm{H}_{7}\right]^{+}, 57$ (44) $\left[\mathrm{C}_{4} \mathrm{H}_{9}\right]^{+}$; HRFAB ${ }^{+} \mathrm{MS}$ : observed $528.2686[\mathrm{M}+\mathrm{H}]^{+}$, (calcd. for $\mathrm{C}_{28} \mathrm{H}_{38} \mathrm{~N}_{3} \mathrm{O}_{7}, 528.2710$ ).

Boc-Gly-Phe-OtBu (2e): Colorless syrup; $[\alpha]+47.2$ (c 1.1, $\mathrm{CHCl}_{3}$ ); IR: 3,414, 3,336, 2,979, 2,934, $1,727,1,671,1,521,1,452,1,369,1,220,1,160,1,044,942,850,743,701 \mathrm{~cm}^{-1} ;{ }^{1} \mathrm{H}-\mathrm{NMR}\left(\mathrm{CDCl}_{3}\right): \delta$ 7.30-7.13 (5H, m, Ar), 6.72 (1H, d, $J=8.0 \mathrm{~Hz}, \mathrm{NH}-\mathrm{Phe}), 5.32$ (1H, dd, $J=4.8,4.8 \mathrm{~Hz}, \mathrm{NH}-\mathrm{Gly}), 4.75$ (1H, dd, $J=14.0,6.0 \mathrm{~Hz}, \mathrm{H \alpha}-\mathrm{Phe}), 3.83$ (1H, dd, $J=16.4,5.2 \mathrm{~Hz}, \mathrm{H \alpha}-\mathrm{Gly}), 3.74$ (1H, dd, $J=16.4$, $5.2 \mathrm{~Hz}, \mathrm{H} \alpha$ '-Gly), 3.08 (2H, d, $J=6.0 \mathrm{~Hz}, \mathrm{H} \beta-\mathrm{Phe}), 1.44$ (9H, s, $\left.\mathrm{CH}_{3}-t \mathrm{Bu}\right), 1.39$ (9H, s, $\left.\mathrm{CH}_{3}-\mathrm{Boc}\right)$; ${ }^{13} \mathrm{C}-\mathrm{NMR}\left(\mathrm{CDCl}_{3}\right) \delta 170.39$ (s, CO-Phe), 169.07 (s, CO-Gly), 155.97 (s, CO-tBu), 136.63 (s, Ar), 129.54 (d, Ar), 128.43 (d, Ar), 127.00 (d, Ar), 82.54 (s, C-Boc), 80.21 (s, C-tBu), 53.66 (d, Ca-Phe),

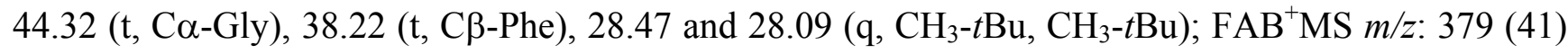
$[\mathrm{M}+\mathrm{H}]^{+}, 323(16)\left[\mathrm{M}+\mathrm{H}-\mathrm{C}_{4} \mathrm{H}_{8}\right]^{+}, 267(100)\left[\mathrm{M}+\mathrm{H}-\mathrm{C}_{4} \mathrm{H}_{8}-\mathrm{C}_{4} \mathrm{H}_{8}\right]^{+}, 223(25)[\mathrm{M}+\mathrm{H}-$ $\left.\mathrm{C}_{7} \mathrm{H}_{10} \mathrm{NO}_{3}\right]^{+}, 166$ (13), 154 (57), 120 (28), 57 (38) $\left[\mathrm{C}_{4} \mathrm{H}_{9}\right]^{+}$; HRFAB ${ }^{+} \mathrm{MS}$ : observed $379.2267[\mathrm{M}+$ $\mathrm{H}]^{+}$, (calcd. for $\mathrm{C}_{20} \mathrm{H}_{31} \mathrm{~N}_{2} \mathrm{O}_{5}, 379.2233$ ). 
Boc-Phe-Phe-OtBu (2f): Colorless crystals; Mp 125-127 ${ }^{\circ} \mathrm{C}$; $[\alpha]+34.1$ (c 1.0, $\left.\mathrm{CHCl}_{3}\right)$; IR, ${ }^{1} \mathrm{H}-\mathrm{NMR}$ and ${ }^{13} \mathrm{C}-\mathrm{NMR}\left(\mathrm{CDCl}_{3}\right)$ are in agreement with previously reported data [32]; $\mathrm{FAB}^{+} \mathrm{MS} m / z$ : 469 (27) $[\mathrm{M}+\mathrm{H}]^{+}, 413(10)\left[\mathrm{M}+\mathrm{H}-\mathrm{C}_{4} \mathrm{H}_{8}\right]^{+}, 357$ (58) $\left[\mathrm{M}+\mathrm{H}-\mathrm{C}_{4} \mathrm{H}_{8}-\mathrm{C}_{4} \mathrm{H}_{8}\right]^{+}, 313$ (78) $\left[\mathrm{M}+\mathrm{H}-\mathrm{C}_{4} \mathrm{H}_{8}-\right.$ $\left.\mathrm{C}_{5} \mathrm{H}_{8} \mathrm{O}_{2}\right]^{+}, 166$ (15), 120 (100), 57 (45) $\left[\mathrm{C}_{4} \mathrm{H}_{9}\right]^{+}$; $\mathrm{HRFAB}^{+} \mathrm{MS}$ : observed $469.2724[\mathrm{M}+\mathrm{H}]^{+}$, (calcd. for $\left.\mathrm{C}_{27} \mathrm{H}_{37} \mathrm{~N}_{2} \mathrm{O}_{5}, 469.2702\right)$.

Boc-Val-Phe-OtBu (2g): Colorless crystals; Mp 112-115 ${ }^{\circ} \mathrm{C}$; $[\alpha]+29.0$ (c 1.01, $\mathrm{CHCl}_{3}$ ); IR: 3,337, 3,282, 2,972, 2,936, 2,874, 1,733, 1,689, 1,659, 1,525, 1,457, 1,371, 1,248, 1,162, 1,020, 848, 752, 696 $\mathrm{cm}^{-1} ;{ }^{1} \mathrm{H}-\mathrm{NMR}\left(\mathrm{CDCl}_{3}\right): \delta$ 7.30-7.15 $(5 \mathrm{H}, \mathrm{m}, \mathrm{Ar}), 6.50(1 \mathrm{H}, \mathrm{d}, J=7.2 \mathrm{~Hz}, \mathrm{NH}-\mathrm{Phe}), 5.16(1 \mathrm{H}, \mathrm{d}$, $J=8.8 \mathrm{~Hz}, \mathrm{NH}-\mathrm{Val}), 4.74(1 \mathrm{H}, \mathrm{dd}, J=14.0,6.4 \mathrm{~Hz}, \mathrm{H \alpha}-\mathrm{Phe}), 3.94$ (1H, dd, $J=8.4,6.8 \mathrm{~Hz}, \mathrm{H} \alpha-\mathrm{Val})$, $3.08(2 \mathrm{H}, \mathrm{dd}, J=6.4,4.4 \mathrm{~Hz}, \mathrm{H} \beta-\mathrm{Phe}), 2.09$ (1H, m, H $\beta-\mathrm{Val}), 1.45$ (9H, s, $\left.\mathrm{CH}_{3}-\mathrm{tBu}\right), 1.38(9 \mathrm{H}, \mathrm{s}$, $\mathrm{CH}_{3}$-Boc), $0.93(3 \mathrm{H}, \mathrm{d}, J=6.8 \mathrm{~Hz}, \mathrm{H} \gamma-\mathrm{Val}), 0.88\left(3 \mathrm{H}, \mathrm{d}, J=6.4 \mathrm{~Hz}, \mathrm{H} \gamma\right.$-Val); ${ }^{3} \mathrm{C}-\mathrm{NMR}\left(\mathrm{CDCl}_{3}\right) \delta$ 171.16 (s, CO-Val), 170.46 (s, CO-Phe), 155.79 (s, CO-Boc), 136.10 (s, Ar), 129.57 (d, Ar), 128.45 (d,

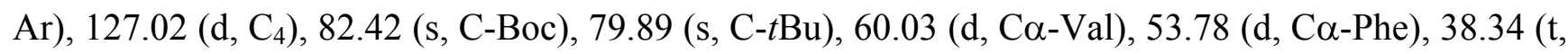

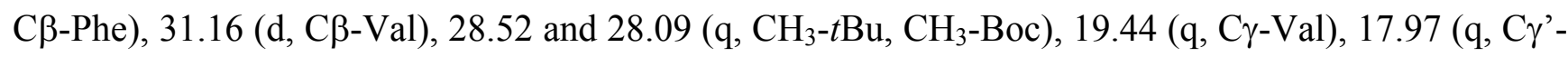
Val); FAB ${ }^{+} \mathrm{MS} m / z$ : 421 (59) $[\mathrm{M}+\mathrm{H}]^{+}, 365$ (17) $\left[\mathrm{M}+\mathrm{H}-\mathrm{C}_{4} \mathrm{H}_{8}\right]^{+}, 309$ (100) $\left[\mathrm{M}+\mathrm{H}-\mathrm{C}_{4} \mathrm{H}_{8}-\mathrm{C}_{4} \mathrm{H}_{8}\right]^{+}$, 265 (95) $\left[\mathrm{M}+\mathrm{H}-\mathrm{C}_{4} \mathrm{H}_{8}-\mathrm{C}_{5} \mathrm{H}_{8} \mathrm{O}_{2}\right]^{+}, 166$ (30), 120 (60), 72 (53), 57 (48) $\left[\mathrm{C}_{4} \mathrm{H}_{9}\right]^{+}$; HRFAB ${ }^{+} \mathrm{MS}$ : observed $421.2666[\mathrm{M}+\mathrm{H}]^{+}$, (calcd. for $\mathrm{C}_{23} \mathrm{H}_{37} \mathrm{~N}_{2} \mathrm{O}_{5}, 421.2702$ ).

Boc-Gly-Val-OtBu (2h): Colorless oil; $[\alpha]+23.8$ (c 1.02, $\mathrm{CHCl}_{3}$ ); IR: 3,333, 2,975, 2,935, 2,878, $1,726,1,671,1,524,1,458,1,391,1,369,1,281,1,251,1,166,1,052,943,848,786 \mathrm{~cm}^{-1} ;{ }^{1} \mathrm{H}-\mathrm{NMR}$ $\left(\mathrm{CDCl}_{3}\right) \delta 6.80(1 \mathrm{H}, \mathrm{bs}, \mathrm{NH}-\mathrm{Val}), 5.48(1 \mathrm{H}, \mathrm{bd}, J=5.6 \mathrm{~Hz}, \mathrm{NH}-\mathrm{Gly}), 4.46(1 \mathrm{H}, \mathrm{dd}, J=9.2,4.4 \mathrm{~Hz}$,

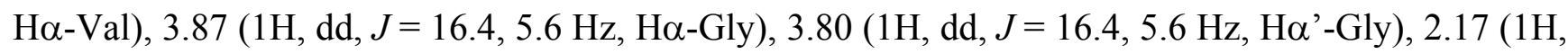
hd, $J=7.2,4.4 \mathrm{H} \beta-\mathrm{Val}), 1.47\left(9 \mathrm{H}, \mathrm{s}, \mathrm{CH}_{3} \mathrm{Boc}\right), 1.46\left(9 \mathrm{H}, \mathrm{s}, \mathrm{CH}_{3} t \mathrm{Bu}\right), 0.94(3 \mathrm{H}, \mathrm{d}, J=7.2 \mathrm{~Hz}, \mathrm{H} \gamma-$ Val), 0.89 (3H, d, $J=7.2 \mathrm{~Hz}, \mathrm{H} \gamma$ '-Val); ${ }^{13} \mathrm{C}-\mathrm{NMR}\left(\mathrm{CDCl}_{3}\right) \delta 170.95$ (s, CO-Val), 169.52 (s, CO-Gly),

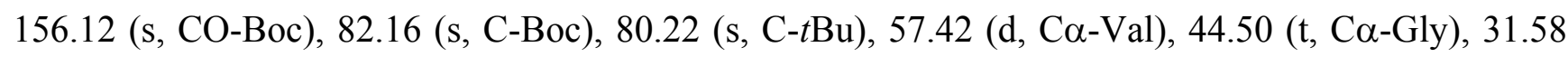

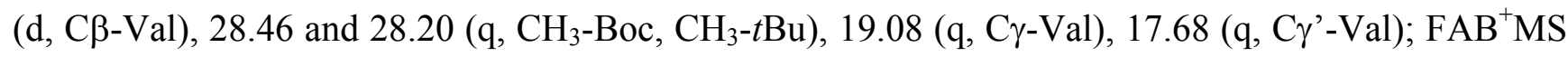
$m / z: 331(28)[\mathrm{M}+\mathrm{H}]^{+}, 275(18)\left[\mathrm{M}+\mathrm{H}-\mathrm{C}_{4} \mathrm{H}_{8}\right]^{+}, 219(100)\left[\mathrm{M}+\mathrm{H}-\mathrm{C}_{4} \mathrm{H}_{8}-\mathrm{C}_{4} \mathrm{H}_{8}\right]^{+}, 175(20)[\mathrm{M}+$ $\left.\mathrm{H}-\mathrm{C}_{4} \mathrm{H}_{8}-\mathrm{C}_{5} \mathrm{H}_{8} \mathrm{O}_{2}\right]^{+}, 72(17)\left[\mathrm{C}_{4} \mathrm{H}_{8} \mathrm{O}\right]^{+}, 57(20)\left[\mathrm{C}_{4} \mathrm{H}_{9}\right]^{+}$; HRFAB ${ }^{+} \mathrm{MS}$ : observed $331.2251[\mathrm{M}+\mathrm{H}]^{+}$, (calcd. for $\mathrm{C}_{16} \mathrm{H}_{31} \mathrm{~N}_{2} \mathrm{O}_{5}, 331.2233$ ).

Boc-Phe-Val-OtBu (2i): White solid; Mp 119-121 ${ }^{\circ} \mathrm{C} ;[\alpha]_{\mathrm{D}}{ }^{\mathrm{Hg}}(365 \mathrm{~nm})+9.0\left(\mathrm{c} 0.5, \mathrm{CHCl}_{3}\right)$; IR: 3,327, $2,975,2,933,1,735,1,687,1,651,1,538,1,367,1,252,1,165,1,025,855 \mathrm{~cm}^{-1} ;{ }^{1} \mathrm{H}-\mathrm{NMR}\left(\mathrm{CDCl}_{3}\right) \delta$ $7.32-7.10(5 \mathrm{H}, \mathrm{Ar}), 6.56(1 \mathrm{H}, \mathrm{d}, J=8.4 \mathrm{~Hz}, \mathrm{NH}-\mathrm{Phe}), 5.20(1 \mathrm{H}, \mathrm{d}, J=8.0 \mathrm{~Hz}, \mathrm{NH}-\mathrm{Val}), 4.41(1 \mathrm{H}, \mathrm{m}$,

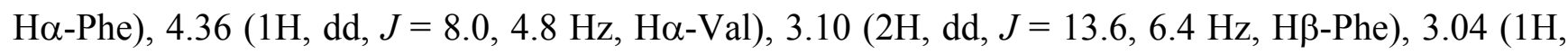
dd, $J=13.6,6.8 \mathrm{~Hz}, \mathrm{H} \beta$ '-Phe), 2.17 (1H, hd, $J=6.8,4.8 \mathrm{H} \beta$-Val), 1.45 (9H, s, $\left.\mathrm{CH}_{3} \mathrm{Boc}\right), 1.41$ (9H, s, $\left.\mathrm{CH}_{3} t \mathrm{Bu}\right), 0.88(3 \mathrm{H}, \mathrm{d}, J=6.8 \mathrm{~Hz}, \mathrm{H} \gamma-\mathrm{Val}), 0.89(3 \mathrm{H}, \mathrm{d}, J=6.8 \mathrm{~Hz}, \mathrm{H} \gamma \cdot-\mathrm{Val}) ;{ }^{13} \mathrm{C}-\mathrm{NMR}\left(\mathrm{CDCl}_{3}\right) \delta$ 171.07 (s, CO-Phe), 170.45 (s, CO-Val), 155.39 (s, CO-Boc), 136.69 (s, C ${ }_{1}$ ), 129.36 (d, $\mathrm{C}_{2}$ and $\mathrm{C}_{6}$ ), $128.58\left(\mathrm{~d}, \mathrm{C}_{3}\right.$ and $\left.\mathrm{C}_{5}\right), 126.85\left(\mathrm{~d}, \mathrm{C}_{4}\right), 81.98$ (s, C-Boc), $80.10(\mathrm{~s}, \mathrm{C}-\mathrm{tBu}), 57.65$ (d, C $\left.\alpha-\mathrm{Val}\right), 55.95(\mathrm{~d}$, $\mathrm{C} \alpha$-Phe), 38.23 (t, C $\beta$-Phe), 31.62 (d, C $\beta$-Val), 28.43 and 28.26 (q, $\mathrm{CH}_{3}-\mathrm{Boc}, \mathrm{CH}_{3}-\mathrm{Bu}$ ), 18.92 (q, $\mathrm{C} \gamma-$ Val), 17.91 (q, C $\left.\gamma^{\prime}-V a l\right) ; \mathrm{FAB}^{+} \mathrm{MS} m / z$ : 421 (46) $[\mathrm{M}+\mathrm{H}]^{+}, 365$ (15) $\left[\mathrm{M}+\mathrm{H}-\mathrm{C}_{4} \mathrm{H}_{8}\right]^{+}, 309$ (100) $[\mathrm{M}+$ 
$\left.\mathrm{H}-\mathrm{C}_{4} \mathrm{H}_{8}-\mathrm{C}_{4} \mathrm{H}_{8}\right]^{+}, 265$ (96) $\left[\mathrm{M}+\mathrm{H}-\mathrm{C}_{4} \mathrm{H}_{8}-\mathrm{C}_{5} \mathrm{H}_{8} \mathrm{O}_{2}\right]^{+}, 120$ (40), 72 (32) $\left[\mathrm{C}_{4} \mathrm{H}_{8} \mathrm{O}\right]^{+}, 57$ (44) $\left[\mathrm{C}_{4} \mathrm{H}_{9}\right]^{+}$; HRFAB ${ }^{+}$MS: observed 421.2688 [M+ H] $]^{+}$, (calcd. for $\mathrm{C}_{23} \mathrm{H}_{37} \mathrm{~N}_{2} \mathrm{O}_{5}, 421.2702$ ).

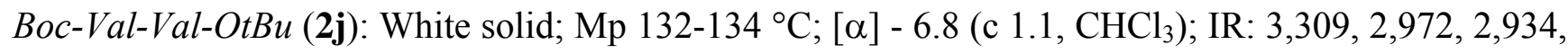
2,888, 1,744, 1,685, 1,651, 1,536, 1,464, 1,372, 1,301, 1,254, 1,219, 1,157, 1,017, $855 \mathrm{~cm}^{-1}$; ${ }^{1} \mathrm{H}-\mathrm{NMR}$ $\left(\mathrm{CDCl}_{3}\right) \delta 6.35(1 \mathrm{H}, \mathrm{d}, J=7.6 \mathrm{~Hz}, \mathrm{NH}-\mathrm{Val}), 5.09(1 \mathrm{H}, \mathrm{d}, J=8.8 \mathrm{~Hz}, \mathrm{NH}-\mathrm{Val}), 4.36(1 \mathrm{H}, \mathrm{dd}, J=8.4$, $4.4 \mathrm{~Hz}, \mathrm{H} \alpha-\mathrm{Val}), 3.86$ (1H, dd, $J=7.6,7.6 \mathrm{~Hz}, \mathrm{H} \alpha-\mathrm{Val}), 2.08$ (2H, m, H $\beta$-Val, H $\beta$-Val), 1.40 (9H, s, $\mathrm{CH}_{3} \mathrm{Boc}$ ), 1.38 (9H, s, $\left.\mathrm{CH}_{3} t \mathrm{Bu}\right), 0.90,0.87,0.86,0.84$ (3H each, d, $J=6.8 \mathrm{~Hz}, \mathrm{H} \gamma-\mathrm{Val}, \mathrm{H} \gamma$ '-Val, H $\gamma-$ Val, H $\gamma^{\prime}-\mathrm{Val}$ ); ${ }^{13} \mathrm{C}-\mathrm{NMR}\left(\mathrm{CDCl}_{3}\right) \delta 171.58$ (s, CO-Val), 170.81 (s, CO-Val), 155.93 (s, CO-Boc),

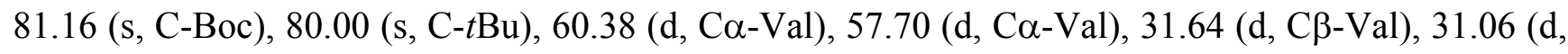
$\mathrm{C} \beta$-Val), 28.56 and 28.29 (q, $\mathrm{CH}_{3}-\mathrm{Boc}, \mathrm{CH}_{3}-\mathrm{Bu}$ ), 19.57, 19.16, 18.21, 17.96 (q, C $\gamma$-Val, $\mathrm{C} \gamma$ '-Val, $\mathrm{C} \gamma-$

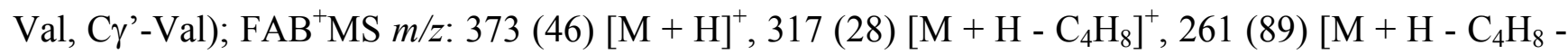
$\left.\mathrm{C}_{4} \mathrm{H}_{8}\right]^{+}, 217$ (94) $\left[\mathrm{M}+\mathrm{H}-\mathrm{C}_{4} \mathrm{H}_{8}-\mathrm{C}_{5} \mathrm{H}_{8} \mathrm{O}_{2}\right]^{+}, 116(26)\left[\mathrm{C}_{5} \mathrm{H}_{10} \mathrm{NO}_{2}\right]^{+}, 72$ (100) $\left[\mathrm{C}_{4} \mathrm{H}_{8} \mathrm{O}\right]^{+}, 57$ (47) $\left[\mathrm{C}_{4} \mathrm{H}_{9}\right]^{+}$; HRFAB ${ }^{+} \mathrm{MS}$ : observed $373.2711[\mathrm{M}+\mathrm{H}]^{+}$, (calcd. for $\mathrm{C}_{19} \mathrm{H}_{37} \mathrm{~N}_{2} \mathrm{O}_{5}, 373.2702$ ).

Boc-Phe-Sar-OtBu (2k): Colorless syrup (73:27 rotamer mixture); [ $\alpha]$ - 22.5 (c 1.58, $\mathrm{CHCl}_{3}$ ); IR: 3,427, 3,322, 2,978, 2,933, 1,741, 1,710, 1,652, 1,491, 1,367, 1,236, 1,164, 1,049, 1,020, 952, 850, 759 and $701 \mathrm{~cm}^{-1}$; ${ }^{1} \mathrm{H}-\mathrm{NMR}\left(\mathrm{CDCl}_{3}\right) \delta 7.30-7.00(5 \mathrm{H}, \mathrm{m}, \mathrm{Ar}), 5.31(1 \mathrm{H}, \mathrm{d}, J=8.8 \mathrm{~Hz}, \mathrm{NH}-\mathrm{Phe}), 4.80$ (1H, dd, $J=15.4,6.6 \mathrm{~Hz}, \mathrm{H \alpha}-\mathrm{Phe}), 3.92$ (1H, d, $J=17.2 \mathrm{~Hz}, \mathrm{H \alpha}-\mathrm{Sar}), 3.84$ (1H, d, $J=17.2 \mathrm{~Hz}, \mathrm{H \alpha}{ }^{\prime}-$ Sar), 2.83 (3H, s, $\mathrm{NCH}_{3}$-Sar), 2.97 (1H, dd, $\left.J=13.6,7.2 \mathrm{~Hz}, \mathrm{H} \beta-\mathrm{Phe}\right), 2.90-2.86$ (1H, m, H $\left.\beta^{\prime}-\mathrm{Phe}\right)$, $1.38\left(9 \mathrm{H}, \mathrm{s}, \mathrm{CH}_{3}-\mathrm{Boc}\right), 1.31\left(9 \mathrm{H}, \mathrm{s}, \mathrm{CH}_{3}-\mathrm{Bu}\right) ;{ }^{13} \mathrm{C}-\mathrm{NMR}\left(\mathrm{CDCl}_{3}\right) \delta 172.11$ (s, CO-Phe), 167.89 (s, CO-Sar), 155.09 (s, CO-Boc), 136.42 (s, C $)_{1}$, 129.65 (d, $\left.\mathrm{C}_{2}, \mathrm{C}_{6}\right), 128.38$ (d, $\left.\mathrm{C}_{3}, \mathrm{C}_{5}\right), 126.85$ (d, $\mathrm{C}_{4}$ ),

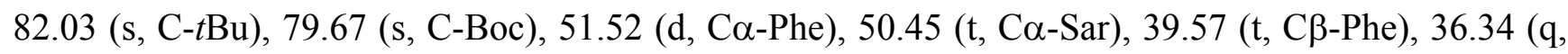
$\mathrm{NCH}_{3}$-Sar), 28.49 and 28.24 (q, $\mathrm{CH}_{3}-\mathrm{Boc}, \mathrm{CH}_{3}-t \mathrm{Bu}$ ); FAB ${ }^{+} \mathrm{MS} m / z: 393$ (27) $[\mathrm{M}+\mathrm{H}]^{+}, 337$ (15) [M + $\left.\mathrm{H}-\mathrm{C}_{4} \mathrm{H}_{8}\right]^{+}, 281(76)\left[\mathrm{M}+\mathrm{H}-2 \mathrm{C}_{4} \mathrm{H}_{8}\right]^{+}, 263(15)\left[\mathrm{M}+\mathrm{H}-\mathrm{C}_{4} \mathrm{H}_{8}-\mathrm{C}_{4} \mathrm{H}_{8} \mathrm{O}\right]^{+}, 237(100)\left[\mathrm{M}+\mathrm{H}-\mathrm{C}_{4} \mathrm{H}_{8}\right.$ - $\left.\mathrm{C}_{5} \mathrm{H}_{8} \mathrm{O}_{2}\right]^{+}, 164(18), 120(81), 90(43)\left[\mathrm{C}_{7} \mathrm{H}_{6}\right]^{+}, 57$ (78) $\left[\mathrm{C}_{4} \mathrm{H}_{9}\right]^{+}$; HRFAB ${ }^{+}$MS: observed 393.2409 [M $+\mathrm{H}]^{+}$, (calcd. for $\mathrm{C}_{21} \mathrm{H}_{33} \mathrm{~N}_{2} \mathrm{O}_{5}, 393.2389$ ).

Boc-Phe-Sar-OMe (2l): Colorless syrup (77:23 rotamer mixture); $[\alpha]+19.1$ (c $0.54, \mathrm{CHCl}_{3}$ ); IR: 3,427, 3,322, 2,977, 2,943, 1,751, 1,708, 1,652, 1,490, 1,407, 1,365, 1,250, 1,212, 1,171, 1,047, 1,021, 751 and $702 \mathrm{~cm}^{-1}$; ${ }^{1} \mathrm{H}-\mathrm{NMR}\left(\mathrm{CDCl}_{3}\right) \delta$ 7.32-7.18 (5H, m, Ar), $5.34(1 \mathrm{H}, \mathrm{d}, J=8.8 \mathrm{~Hz}, \mathrm{NH}-\mathrm{Phe}), 4.88$ $(1 \mathrm{H}, \mathrm{dd}, J=14.8,6.8 \mathrm{~Hz}, \mathrm{H \alpha}-\mathrm{Phe}), 4.14$ (1H, d, $J=17.2 \mathrm{~Hz}, \mathrm{H \alpha}-\mathrm{Sar}), 3.99$ (1H, d, $J=17.2 \mathrm{~Hz}, \mathrm{H}^{\prime}-$ Sar), 3.73 (3H, s, $\left.\mathrm{OCH}_{3}\right), 3.04$ (1H, dd, $\left.J=13.6,7.2 \mathrm{~Hz}, \mathrm{H} \beta-\mathrm{Phe}\right), 2.95$ (1H, dd, $J=13.6,6.4 \mathrm{~Hz}, \mathrm{H} \beta$ 'Phe), 2.87 (3H, s, $\mathrm{NCH}_{3}$-Sar), $1.40\left(9 \mathrm{H}, \mathrm{s}, \mathrm{CH}_{3}\right.$-Boc); ${ }^{13} \mathrm{C}-\mathrm{NMR}\left(\mathrm{CDCl}_{3}\right) \delta 172.35$ (s, CO-Phe), 169.28 (s, CO-Sar), 155.07 (s, CO-Boc), 136.33 (s, Ar), 129.65 (d, Ar), 128.45 (d, Ar), 126.93 (d, C 4 ), 79.81

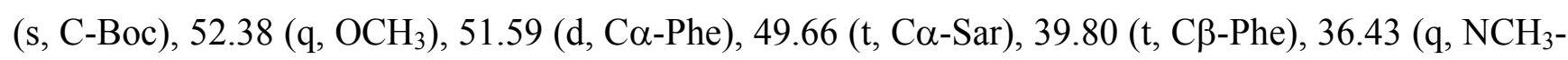
Sar), 28.55 (q, $\left.\mathrm{CH}_{3}-\mathrm{Boc}\right) ; \mathrm{FAB}^{+} \mathrm{MS} m / z: 351(33)[\mathrm{M}+\mathrm{H}]^{+}, 295$ (55) $\left[\mathrm{M}+\mathrm{H}-\mathrm{C}_{4} \mathrm{H}_{8}\right]^{+}, 251$ (100) $[\mathrm{M}+$ $\left.\mathrm{H}-\mathrm{C}_{5} \mathrm{H}_{9} \mathrm{O}_{2}\right]^{+}, 164$ (16), 120 (67), 104 (70) $\left[\mathrm{C}_{4} \mathrm{H}_{10} \mathrm{NO}_{2}\right]^{+}, 57$ (44) $\left[\mathrm{C}_{4} \mathrm{H}_{9}\right]^{+}, 44$ (18); HRFAB ${ }^{+} \mathrm{MS}$ : observed $351.1907[\mathrm{M}+\mathrm{H}]^{+}$, (calcd. for $\mathrm{C}_{18} \mathrm{H}_{27} \mathrm{~N}_{2} \mathrm{O}_{5}, 351.1920$ ). 


\subsection{General procedure for the syntheses of 2,5-diketopiperazines $\mathbf{3 a - 3 \mathbf { k }}$}

Each $N \alpha$-Boc-dipeptidyl ester $(0.25 \mathrm{mmol})$ was dissolved or suspended in water $(1 \mathrm{~mL})$ and heated during 10 minutes at $250{ }^{\circ} \mathrm{C}$ and $150 \mathrm{psi}$, using a monomode CEM Discover microwave apparatus at $250 \mathrm{~W}$. The resulting suspension was filtered through a Hirsch funnel and washed with water $(5 \mathrm{~mL})$, the solid was dried under high vacuum and analyzed without further purification by NMR. Compounds 3h and 3k were water soluble, and in these cases, resulting solutions were lyophilized and the solids purified as indicated in Table 2 and analyzed by NMR.

Cyclo[Val-Orn(Cbz)] (3a): White solid; Mp 202-204 ${ }^{\circ} \mathrm{C}\left\{\right.$ lit [33] 206-208 $\left.{ }^{\circ} \mathrm{C}\right\}$; [ $\alpha$ ] - 26.0 (c 0.27, DMSO) $\{$ lit [33] - 47.4 (c $1 \%)\}$; IR: 3,434, 3,333, 3,201, 3,094, 3,052, 2,965, 2,878, 1,678, 1,531, 1,444, 1,258, 1,142, 1,025, 774, 696, $629 \mathrm{~cm}^{-1}$; ${ }^{1} \mathrm{H}-\mathrm{NMR}$ (DMSO): $\delta 8.14$ (1H, s, NH-Orn), $8.04(1 \mathrm{H}$, s, NH-Val), 7.39-7.26 (6H, m, Ar, NHס-Orn), 5.00 (2H, s, $\left.\mathrm{CH}_{2}-\mathrm{Cbz}\right), 3.81$ (1H, t, $\left.J=4.8 \mathrm{~Hz}, \mathrm{H \alpha}-\mathrm{Orn}\right)$,

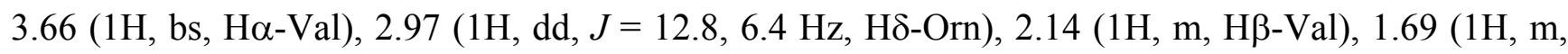

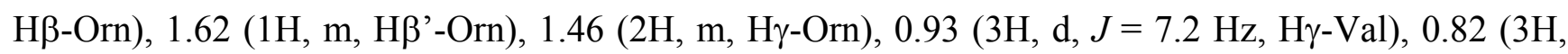
$\mathrm{m}, \mathrm{d}, J=7.2 \mathrm{~Hz}, \mathrm{H} \gamma^{\prime}-\mathrm{Val}$ ); ${ }^{13} \mathrm{C}-\mathrm{NMR}$ (DMSO): $\delta 167.83$ (s, CO-Orn), 166.88 (s, CO-Val), 156.06 (s, CO-Cbz), 137.21 (s, Ar), 128.33 (d, Ar), 127.64 (d, Ar), 65.18 (t, $\mathrm{CH}_{2}-\mathrm{Cbz}$ ), 59.43 (d, Ca-Val), 53.75

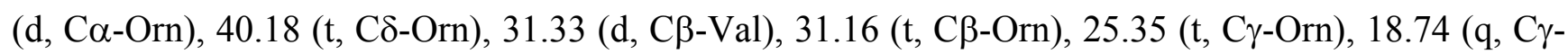
Val), 17.29 (q, C $\gamma^{\prime}-\mathrm{Val}$ ); $\mathrm{FAB}^{+} \mathrm{MS} m / z: 348$ (87) $[\mathrm{M}+\mathrm{H}]^{+}, 307$ (100) $\left[\mathrm{M}+\mathrm{H}-\mathrm{C}_{3} \mathrm{H}_{6}\right]^{+}, 289$ (61), 240 (22) $\left[\mathrm{M}+\mathrm{H}-\mathrm{C}_{7} \mathrm{H}_{8} \mathrm{O}\right]^{+}, 219$ (25) $\left[\mathrm{C}_{12} \mathrm{H}_{15} \mathrm{~N}_{2} \mathrm{O}_{2}\right]^{+}, 214$ (12), 195 (12), 165 (12); HRFAB ${ }^{+}$MS: observed $348.1887[\mathrm{M}+\mathrm{H}]^{+}$, (calcd. for $\mathrm{C}_{18} \mathrm{H}_{26} \mathrm{~N}_{3} \mathrm{O}_{4}, 348.1923$ ).

Cyclo[Val-D-Orn $(\mathrm{Cbz})]$ (3b): White solid; Mp 212-214 ${ }^{\circ} \mathrm{C}$; $[\alpha]+11.7$ (c 0.5, MeOH); IR: 3,347, 3,192, 3,054, 2,962, 1,675, 1,540, 1,460, 1,265, 1,143, 1,031, 852, $696 \mathrm{~cm}^{-1}$; ${ }^{1} \mathrm{H}-\mathrm{NMR}$ (DMSO): $\delta 8.11$

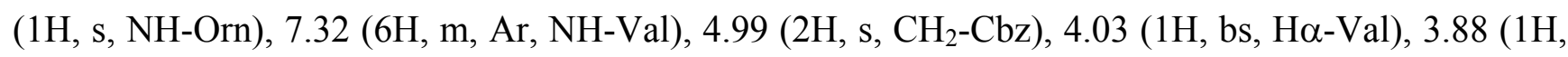

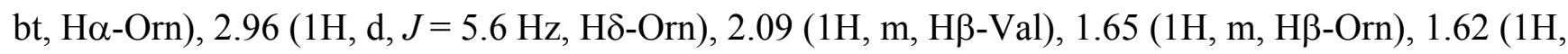

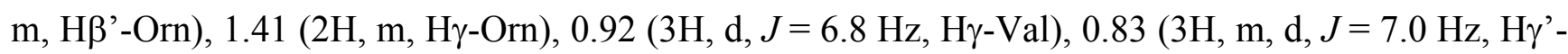
Val); ${ }^{13} \mathrm{C}-\mathrm{NMR}$ (DMSO): $\delta 168.13$ (s, CO-Orn), 167.67 (s, CO-Val), 156.17 (s, CO-Cbz), 137.25 (s,

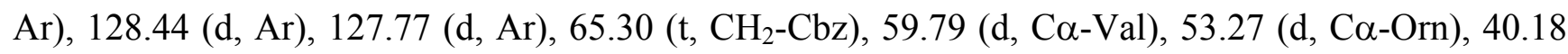

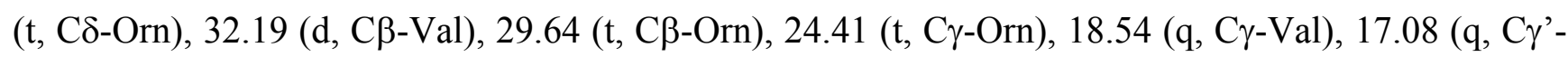
Val); FAB ${ }^{+} \mathrm{MS} m / z: 348$ (3) [M + H] $]^{+}, 219$ (5), 154 (12), 130 (30), 107 (8), 91 (100), 85 (28), 72 (25); HRFAB ${ }^{+}$MS: observed $348.1949[\mathrm{M}+\mathrm{H}]^{+}$, (calcd. for $\mathrm{C}_{18} \mathrm{H}_{26} \mathrm{~N}_{3} \mathrm{O}_{4}, 348.1923$ ).

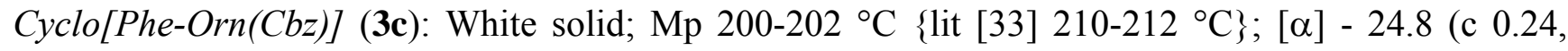
DMSO) \{lit [33] - 12.9 (c $1 \%$ ) ; IR: 3,322, 3,189, 3,039, 2,965, 2,894, 1,669, 1,532, 1,458, 1,338, 1,246, 1,134, 1,101, 1,016, 852, 753, $669 \mathrm{~cm}^{-1}$; ${ }^{1} \mathrm{H}-\mathrm{NMR}$ (DMSO): $\delta 8.16(1 \mathrm{H}, \mathrm{s}, \mathrm{NH}-\mathrm{Phe}), 8.05(1 \mathrm{H}$,

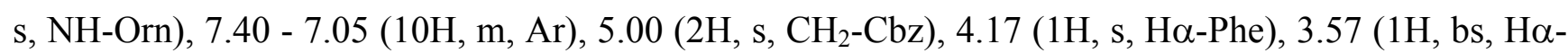
Orn), $3.13(1 \mathrm{H}, \mathrm{dd}, J=13.6,4.0 \mathrm{~Hz}, \mathrm{H} \beta-\mathrm{Phe}), 2.83$ (1H, dd, $J=13.6,5.2 \mathrm{~Hz}, \mathrm{H} \beta$ '-Phe), 2.68 (1H, dd,

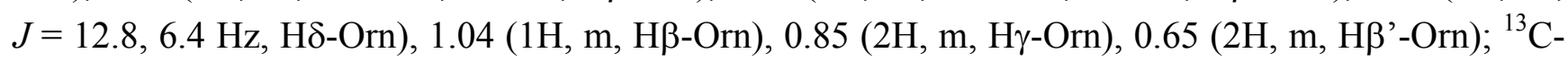
NMR (DMSO): $\delta 166.70$ (s, CO-Orn), 166.00 (s, CO-Phe), 155.79 (s, CO-Cbz), 137.16 (s, Ar-Cbz), 135.92 (s, Ar), 130.16 (d, Ar), 128.27 (d, Ar), 127.92 (d, Ar), 127.69 (d, Ar), 126.57 (d, Ar), 65.10 (t,

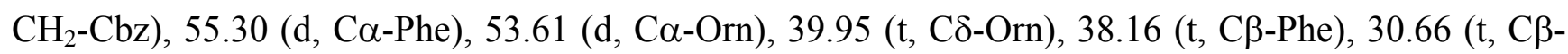

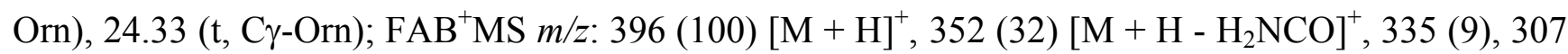


(53) $\left[\mathrm{M}+\mathrm{H}-\mathrm{C}_{7} \mathrm{H}_{6}\right]^{+}, 289$ (32) $\left[\mathrm{M}+\mathrm{H}-\mathrm{C}_{7} \mathrm{H}_{7} \mathrm{O}\right]^{+}, 243(16)\left[\mathrm{M}+\mathrm{H}-\mathrm{C}_{14} \mathrm{H}_{16} \mathrm{NO}_{2}\right]^{+}, 219$ (18) $[\mathrm{M}+\mathrm{H}-$ $\left.\mathrm{C}_{10} \mathrm{H}_{12} \mathrm{NO}_{2}\right]^{+}, 165$ (14); HRFAB ${ }^{+} \mathrm{MS}$ : observed $396.1949[\mathrm{M}+\mathrm{H}]^{+}$, (calcd. for $\mathrm{C}_{22} \mathrm{H}_{26} \mathrm{~N}_{3} \mathrm{O}_{4}$, 396.1923).

Cyclo(Phe-Gly) (3e): White solid; Mp 266-268 ${ }^{\circ} \mathrm{C}\left\{\right.$ lit [34] 271-273 $\left.{ }^{\circ} \mathrm{C}\right\} ;[\alpha]+26.6$ (c 0.95, DMSO) $\{$ lit [34] + 7.3 (c 0.95, DMSO)\}; IR: 3,426, 3,190, 3,057, 2,977, 2,921, 2,878, 1,676, 1,462, 1,332, 1,086, 1,004, 847, 794, 758, $702 \mathrm{~cm}^{-1} ;{ }^{1} \mathrm{H}$ - and ${ }^{13} \mathrm{CNMR}$ (DMSO) are in agreement with previously reported data [35]; $\mathrm{FAB}^{+} \mathrm{MS} m / z$ : 205 (13) $[\mathrm{M}+\mathrm{H}]^{+}, 169$ (15), 154 (24), 130 (43) [M + H - $\left.\mathrm{C}_{4} \mathrm{H}_{4}\right]^{+}, 85$ (100) $\left[\mathrm{C}_{3} \mathrm{H}_{3} \mathrm{NO}_{2}\right]^{+}$; HRFAB ${ }^{+} \mathrm{MS}$ : observed 205.1058 [M+ H] $]^{+}$, (calcd. for $\mathrm{C}_{11} \mathrm{H}_{13} \mathrm{~N}_{2} \mathrm{O}_{2}, 205.0977$ ).

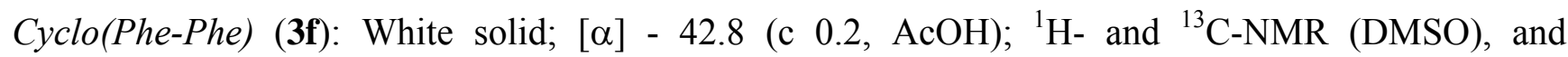
$\mathrm{HRFAB}^{+} \mathrm{MS}$ are in agreement with previously reported data [36]; IR: 3,440, 3,318, 3,198, 3,056, 2,970, 2,929, 1,669, 1,458, 1,338, 1,197, 1,088, 1,013, 759 and $700 \mathrm{~cm}^{-1}$.

Cyclo(Val-Phe) (3g): White solid; Mp 264-266 ${ }^{\circ} \mathrm{C}\left\{\right.$ lit [36] 263-265 $\left.{ }^{\circ} \mathrm{C}\right\} ;[\alpha]-66.0$ (c 0.28, DMSO) \{lit [37] - 64 (c 0.2 AcOH); [34] - 43.3 (c 0.27 DMSO)\}; IR: 3,440, 3,316, 3,192, 3,056, 2,967, 2,885, $1,668,1,454,1,341,1,090,859,758,699, \mathrm{~cm}^{-1} ;{ }^{1} \mathrm{H}-$ and ${ }^{13} \mathrm{C}-\mathrm{NMR}$ (DMSO) are in agreement with previously reported data [34,37]; $\mathrm{FAB}^{+} \mathrm{MS} m / z: 247(100)[\mathrm{M}+\mathrm{H}]^{+}, 219(13)[\mathrm{M}+\mathrm{H}-\mathrm{CO}]^{+}, 217$ (10), 203 (7), 165 (5); HRFAB ${ }^{+}$MS: observed $247.1446[\mathrm{M}+\mathrm{H}]^{+}$, (calcd. for $\mathrm{C}_{14} \mathrm{H}_{19} \mathrm{~N}_{2} \mathrm{O}_{2}, 247.1447$ ).

Cyclo(Val-Gly) (3h): White solid; Mp 210-212 ${ }^{\circ} \mathrm{C}\left\{\right.$ lit [38] $\left.256{ }^{\circ} \mathrm{C}\right\} ;$ [ $\left.\alpha\right]+32.9$ (c 0.46, $\left.\mathrm{H}_{2} \mathrm{O}\right)$ \{lit [38] + $\left.23.7\left(\mathrm{c} 1.0, \mathrm{H}_{2} \mathrm{O}\right)\right\}$; IR: 3,431, 3,199, 3,055, 2,925, 2,859, 1,670, 1,461, 1,381, 1,346, 1,109, 1,047, 808, $621 \mathrm{~cm}^{-1}$; ${ }^{1} \mathrm{H}$ - and ${ }^{13} \mathrm{C}-\mathrm{NMR}$ (DMSO) are in agreement with previously reported data [38,39]; $\mathrm{FAB}^{+} \mathrm{MS} m / z$ : 157 (94) $[\mathrm{M}+\mathrm{H}]^{+}, 154$ (100) [M - $\left.\mathrm{H}_{2}\right], 136$ (78), 120 (12), 107 (25), $85(58)\left[\mathrm{C}_{5} \mathrm{H}_{9} \mathrm{O}\right]^{+}$, 77 (25), 55 (32), $43(27)\left[\mathrm{C}_{3} \mathrm{H}_{7}\right]^{+}, 41$ (25); $\mathrm{HRFAB}^{+} \mathrm{MS}$ : observed $157.0957[\mathrm{M}+\mathrm{H}]^{+}$, (calcd. for $\left.\mathrm{C}_{7} \mathrm{H}_{13} \mathrm{~N}_{2} \mathrm{O}_{2}, 157.0957\right)$.

Cyclo(Val-Val) (3j): Colorless needless; Mp $268{ }^{\circ} \mathrm{C}$ with sublimation $\left\{\right.$ lit [40] $\left.268{ }^{\circ} \mathrm{C}\right\}$; [ $\alpha$ ] -54.8 (c $0.5, \mathrm{AcOH})\{$ lit [15] - 62 (c 0.5, AcOH); IR: 3,434, 3,325, 3,193, 3,100, 3,057, 2,966, 2,880, 1,664,

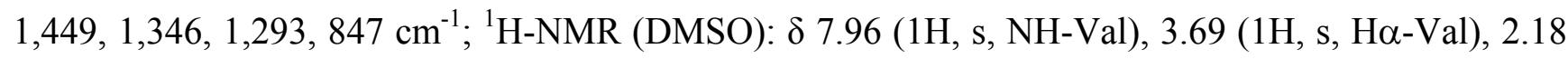
$(1 \mathrm{H}, \mathrm{m}, \mathrm{H} \beta-\mathrm{Val}), 0.95(3 \mathrm{H}, \mathrm{d}, J=7.6 \mathrm{~Hz}, \mathrm{H} \gamma-\mathrm{Val}), 0.83\left(3 \mathrm{H}, \mathrm{d}, J=7.6 \mathrm{~Hz}, \mathrm{H} \gamma\right.$ '-Val); ${ }^{13} \mathrm{C}-\mathrm{NMR}$

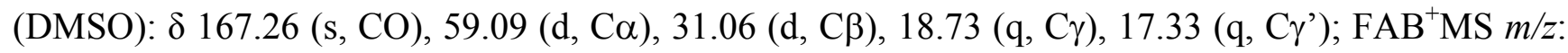
$199(100)[\mathrm{M}+\mathrm{H}]^{+}, 197$ (19), 169 (8); HRFAB ${ }^{+} \mathrm{MS}$ : observed $199.1463[\mathrm{M}+\mathrm{H}]^{+}$, (calcd. for $\left.\mathrm{C}_{10} \mathrm{H}_{19} \mathrm{~N}_{2} \mathrm{O}_{2}, 199.1447\right)$.

Cyclo(Sar-Phe) (3k): Colorless needles; Mp 180-183 ${ }^{\circ} \mathrm{C}\left\{\right.$ lit [41] 184-185 $\left.{ }^{\circ} \mathrm{C}\right\} ;[\alpha]+56.0$ (c 1.01, $\mathrm{MeOH})\{$ lit [41] + 47.5 (c $2.3 \mathrm{MeOH})\}$; IR: 3,440, 3,253, 2,963, 2,929, 2,895, 1,656, 1,470, 1,441, 1,322, 1,102, 1,033, 870, 754, 699, $\mathrm{cm}^{-1} ;{ }^{1} \mathrm{H}-\mathrm{NMR}\left(\mathrm{CDCl}_{3}\right): \delta 7.60(1 \mathrm{H}, \mathrm{bs}, \mathrm{NH}-\mathrm{Phe}), 7.35-7.16(5 \mathrm{H}$,

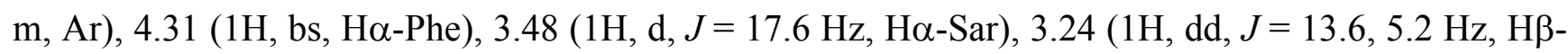
Phe), 3.07 (1H, dd, $J=13.6,4.4$ Hz, Hß'-Phe), 2.81 (3H, s, $\mathrm{NCH}_{3}$-Sar), 2.79 (1H, d, $J=17.6 \mathrm{~Hz}, \mathrm{H \alpha}$ 'Sar); ${ }^{13} \mathrm{C}-\mathrm{NMR}\left(\mathrm{CDCl}_{3}\right): \delta 166.32$ (s, CO-Sar), 165.52 (s, CO-Phe), 134.99 (s, Ar), 130.07 (d, Ar),

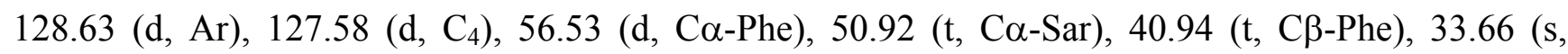


$\mathrm{NCH}_{3}$-Sar); FAB ${ }^{+} \mathrm{MS} m / z: 219$ (100) $[\mathrm{M}+\mathrm{H}]^{+}, 154(50)[\mathrm{M}+\mathrm{H}-\mathrm{CO}]^{+}, 136(56), 91(35)\left[\mathrm{C}_{7} \mathrm{H}_{7}\right]^{+}, 73$ (58); HRFAB ${ }^{+} \mathrm{MS}$ : observed 219.1134 [M+ H] $]^{+}$, (calcd. for $\left.\mathrm{C}_{12} \mathrm{H}_{15} \mathrm{~N}_{2} \mathrm{O}_{2}, 219.1134\right)$.

\section{Conclusions}

Optically pure cis-DKPs could be synthesized in one-pot from the corresponding $N \alpha$-Bocdipeptidyl-tert-butyl esters in water under microwave irradiation for ten minutes. Employing these conditions, the tert-butoxy group is efficiently removed, leading to cyclization in excellent yields. This is the first protocol for ring closures using $N \alpha$-Boc-dipeptidyl-tert-butyl esters. The trans-DKP fragment present in the natural product 1 was synthesized in quantitative yield. The reaction is rapid, secure, environmentally friendly and highly efficient.

\section{Acknowledgements}

This work was financially supported by CONACyT (Grants number 79584-Q and 2006-C01-LN56431). Lemuel Pérez-Picaso thanks CONACyT for a doctoral fellowship (number 181754). We are grateful to Dr. A. Berenice Aguilar-Guadarrama, Dr. Blanca Domínguez Mendoza, Dr. Diana Gabriela Vargas Pineda, Ing. Victoria Labastida, and T. C. María Medina Pastor for technical assistance.

\section{References and Notes}

1. Wipf, P. Synthetic studies of biologically active marine cyclopeptides. Chem. Rev. 1995, 95, 2115-2134.

2. Amidon, G.L.; Lee, H.J. Absorption of peptide and peptidomimetic drugs. Annu. Rev. Pharmacol. Toxicol. 1994, 34, 321-341.

3. Liu, S.; Gu, W.; Lo, D.; Ding, X.; Ujiki, M.; Adrian, T.E.; Soff, G.A.; Silverman, R.B. N-Methylsansalvamide A peptide analogues. Potent new antitumor agents. J. Med. Chem. 2005, 48, 3630-3638.

4. Prasad, C. Bioctive cyclic dipeptides. Peptides 1995, 16, 151-164.

5. Houston, D.R.; Synstad, B.; Eijsink, V.G.H.; Stark, M.J.R.; Eggleston, I.M.; van Aalten, D.M.F. Structure-based exploration of cyclic dipeptide chitinase inhibitors. J. Med. Chem. 2004, 47, 5713-5720.

6. Martins, M.B.; Carvalho, I. Diketopiperazines: biological activity and synthesis. Tetrahedron 2007, 63, 9923-9932.

7. Graz, C.J.M.; Grant, G.D.; Brauns, S.C.; Hunt, A.; Jamie, H.; Milne, P.J. Cyclic dipeptides in the induction of maturation for cancer therapy. J. Pharm. Pharmacol. 2000, 52, 75-82.

8. Prakash, K.R.C.; Tang, Y.; Kozikowski, A.P.; Flippen-Anderson, J.L.; Knoblach, S.M.; Faden, A.I. Synthesis and biological activity of novel neuroprotective diketopiperazines. Bioorg. Med. Chem. 2002, 10, 3043-3048.

9. Lambert, J.N.; Mitchell, J.P.; Roberts, K.D. The synthesis of cyclic peptides. J. Chem. Soc. Perkin Trans. I 2001, 471-484.

10. Rajappa, S.; Natekar, M.V. Piperazine-2,5-diones and related lactam ethers. Adv. Het. Chem. 1993, 57, 187-289. 
11. Fischer, P.M.J. Diketopiperazines in peptide and combinatorial chemistry. J. Pept. Sci. 2003, 9, 9-35.

12. Dinsmore, C.J.; Beshore, D.C. Recent advances in the synthesis of diketopiperazines. Tetrahedron 2002, 58, 3297-3312.

13. Rodionov, I.L.; Rodionova, L.N.; Baidakova, L.K.; Romashko, A.M.; Balashova, T.A.; Ivanov, V.T. Cyclic dipeptides as building blocks for combinatorial libraries. Part 2: Synthesis of bifunctional diketopiperazines. Tetrahedron 2002, 58, 8515-8523.

14. Fischer, E. Synthese von polypeptiden. Chem. Ber. 1906, 39, 2893-2931.

15. Ueda, T.; Saito, M.; Kato, T.; Izumiya, N. Facile Synthesis of Cyclic Dipeptides and Detection of Racemization. Bull. Chem. Soc. Jpn. 1983, 56, 568-572.

16. Suzuki, K.; Sasaki, Y.; Endo, N.; Mihara, Y. Acetic acid-catalized diketopiperazine synthesis. Chem. Pharm. Bull. 1981, 29, 233-237.

17. Nitecki, D.E.; Halpern, B.; Westley, J.W. Simple route to sterically pure dioxopiperazines. J. Org. Chem. 1968, 33, 864-866.

18. Eriksson, J.; Arvidsson, P.I.; Davidsson, O. Solution structure of a dilithiumamide/diethylzinc heterocomplex that catalyzes asymmetric alkylation reactions. Chem. Eur. J. 1999, 5, 2356-2361.

19. Lee, S.; Kanmera, T.; Aoyagi, H.; Izumiya, N. Cyclic peptides. VI. Asymmetric hydrogenation of dehydroalanine or dehydroaminobutanoic acid residue in cyclodipeptides. Int. J. Pept. Protein Res. 1979, 13, 207-217.

20. Kappe, C.O.; Dallinger, D. Controlled microwave heating in modern organic synthesis: highlights from the 2004-2008 literature. Mol. Divers. 2009, 13, 71-193.

21. Kappe, C.O.; Dallinger, D.; Murphree, S.S. Practical microwave synthesis for organic chemists: Strategies, Instruments, and Protocols, 1st ed.; Wiley-VCH: Darmstadt, Germany, 2009.

22. Santagada, V., Fiorino, F.; Perissutti, E.; Severino, B.; Terracciano, S.; Cirino, G.; Caliendo, G. A convenient strategy of dimerization by microwave heating and using 2,5-diketopiperazine as scaffold. Tetrahedron Lett. 2003, 44, 1145-1148.

23. López-Cobeñas, A.; Cledera, P.; Sánchez, J.D.; Pérez-Contreras, R.; López-Alvarado, P.; Ramos, M.T.; Avendaño, C.; Menéndez, J.C. Solvent-Free, Efficient Synthesis of 2,5-Piperazinediones from Boc-protected dipeptide esters under microwave irradiation. Synlett 2005, 7, 1158-1160.

24. Tullberg, M.; Grøtli, M.; Luthman, K. Efficient synthesis of 2,5-diketopiperazines using microwave assisted heating. Tetrahedron 2006, 62, 7484-7491.

25. Jam, F.; Tullberg, M.; Luthman, K.; Grøtli, M. Microwave assisted synthesis of spiro-2,5diketopiperazines. Tetrahedron 2007, 63, 9881-9889.

26. Fischer, P.M.; Solbakken, M.; Undheim, K. Solution synthesis of a dimeric pentapeptide: diketopiperazine cyclisation of Glu-Asp dipeptide esters and Asp-racemisation during segment condensation. Tetrahedron 1994, 50, 2277-2288.

27. Besser, D.; Greiner, G.; Reissmann, S. Side reaction with N-carboxymethyl amino acids in the synthesis of lactone cyclized peptides. Lett. Pept. Sci. 1998, 5, 299-303.

28. Pérez-Picaso, L.; Rios, M.Y.; Hernández, A.N.; Martínez, J. ${ }^{1} \mathrm{H}$ and ${ }^{13} \mathrm{C}$ assignments of cyclo $[\mathrm{N}-$ (Lys-Phe)-Orn-Val], a semicyclic imide tetrapeptide from Burkholderia cepacia. Magn. Reson. Chem. 2006, 44, 959-961. 
29. Pandey, S.K.; Awasthi, K.K.; Saxena, A.K. Microwave assisted stereospecific synthesis of (S)-3-substituted 2,3,6,7,12,12a-hexahydropyrazino[1',2':1,6]pyrido[3,4-b]indole-1,4-diones. Tetrahedron 2001, 57, 4437-4432.

30. Kobayashi, N.; Higuchi, T.; Urano, Y.; Kikuchi, K.; Hirobe, M.; Nagano, T. Dipeptides Containing L-arginine analogs: New isozyme-selective inhibitors of nitric oxide synthase. Biol. Pharm. Bull. 1999, 22, 936-940.

31. Shen, H.Y.; Tian, G.L.; Ye, Y.H.; Wang, J. Non-coded amino acids as acyl donor substrates for peptide bond formation catalyzed by thermoase in toluene. J. Mol. Catal. B-Enzym. 2005, 37, 26-29.

32. Dineen, T.A.; Zajac, M.A.; Myers, A.G. Efficient transamidation of primary carboxamides by in situ activation with N,N-dialkylformamide dimethyl acetals. J. Am. Chem. Soc. 2006, 128, 16406-16409.

33. Izumiya, N.; Kato, T.; Fujita, Y.; Ohno, M.; Kondo, M. Studies of peptide antibiotics, I. Dipeptide anhydrides as models of cyclic peptide antibiotics. Bull. Chem. Soc. Jpn. 1964, 37, 1809-1816.

34. López-Cobeñas, A.; Cledera, P.; Sánchez, J.D.; López-Alvarado, P.; Ramos, M.T.; Avendaño, C.; Menéndez, J.C. Microwave-assisted synthesis of 2,5-piperazinediones under solvent-free conditions. Synthesis 2005, 19, 3412-3422.

35. Huang, H.; She, Z.; Lin, Y.; Vrijmoed, L.L.P.; Lin, W. Cyclic Peptides from an Endophytic Fungus Obtained from a Mangrove Leaf (Kandelia candel). J. Nat. Prod. 2007, 70, 1696-1699.

36. Joshi, K.B., Verma, S. Participation of aromatic side chains in diketopiperazine ensembles. Tetrahedron Lett. 2008, 49, 4231-4234.

37. Tullberg, M.; Luthman, K.; Grøtli, M. Microwave-assisted solid-phase synthesis of 2,5diketopiperazines: Solvent and resin dependence. J. Comb. Chem. 2006, 8, 915-922.

38. Bull, S.D.; Davies, S.G.; Moss, W.O. Practical synthesis of Schöllkopf's bis-lactim ether chiral auxiliary: (3S)-3,6-dihydro-2,5-dimethoxy-3-isopropyl-pyrazine. Tetrahedron: Asym. 1998, 9, 321-327.

39. Cledera, P.; Avendaño, C.; Menéndez, J.C. Comparative study of synthetic approaches to 1arylmethylenepyrazino[2,1-b]quinazoline-3,6-diones. Tetrahedron 1998, 54, 12349-12360.

40. Tanihara, M.; Hiza, T.; Imanishi, Y.; Higashimura, T. Solution conformation of cyclic dipeptides having aliphatic side chains. Bull. Chem. Soc. Jpn. 1983, 56, 1155-1160.

41. Lucente, G.; Pinnen, F.; Zanotti, G. Cyclization of activated N-benzyloxycarbonyl-tripeptides. Tetrahedron Lett. 1978, 11, 1009-1012.

Sample Availability: Samples of the compounds $\mathbf{2 a - 2 l}$ and 3a-3k are available from the authors.

(C) 2009 by the authors; licensee Molecular Diversity Preservation International, Basel, Switzerland. This article is an open-access article distributed under the terms and conditions of the Creative Commons Attribution license (http://creativecommons.org/licenses/by/3.0/). 